\title{
Hierarchical scaling law for the strength of composite fibre bundles
}

\author{
Soraia Pimenta*, Silvestre T. Pinho \\ Department of Aeronautics, South Kensington Campus, Imperial College London. London SW7 $2 A Z$, \\ United Kingdom
}

\begin{abstract}
This paper presents an analytical model for size effects on the longitudinal tensile strength of composite fibre bundles. The strength of individual fibres is modelled by a Weibull distribution, while the matrix (or fibre-matrix interface) is represented through a perfectly-plastic shear-lag model. A probabilistic analysis of the failure process in hierarchical bundles (bundles of bundles) is performed, so that a scaling law relating the strength distributions and characteristic lengths of consecutive bundle levels is derived. An efficient numerical scheme (based on asymptotic limits) is proposed, hence couponsized bundle strength distributions are obtained almost instantaneously. Parametric studies show that both fibre and matrix properties are critical for bundle strength; model predictions at different scales are validated against experimental results available in the literature.

Keywords: A. strengthening and mechanisms, B. fibre-reinforced composite material, C. probability and statistics, Size effects.
\end{abstract}

\section{Introduction}

Size effects on the strength of composite materials are widely reported in the literature, but a universally accepted modelling strategy is still to be developed (Wisnom, 1999); this represents a significant challenge for the design of large structures. This paper presents a model for size effects on the longitudinal tensile strength of UniDirectional (UD) Fibre-Reinforced Polymers (FRPs), based on the stochastic variability of fibre strength and the definition of hierarchical fibre-matrix bundles.

\footnotetext{
*Corresponding author.

Email address: soraia.pimenta@imperial.ac.uk (Soraia Pimenta)
}

Pimenta S, Pinho ST (2013). Hierarchical scaling law for the strength of composite fibre bundles. Journal of the Mechanics and Physics of Solids 61, 1337-1356.DOI:10.1016/j.jmps.2013.02.004 


\begin{tabular}{|c|c|c|}
\hline \multicolumn{3}{|c|}{ Nomenclature } \\
\hline \multicolumn{2}{|c|}{ Uppercase variables } & Superscripts \\
\hline$A$ & cross sectional area & f fibre \\
\hline$C$ & perimeter & [i] bundle level \\
\hline & stress-concentrations strength parameter & $\infty$ remote \\
\hline $\mathrm{CoV}_{X}$ & coefficient of variation of strength & \\
\hline & failure probability (strength distribution) & \\
\hline$S$ & survival probability & Subscripts \\
\hline$V$ & volume fraction & $0 \quad$ Weibull scale parameter \\
\hline & longitudinal tensile strength & free edges boundary \\
\hline Lower & rcase variables & c control length \\
\hline$\phi$ & diameter & effective recovery length \\
\hline$i$ & bundle level & linear stress concentrations state \\
\hline$k$ & stress concentrations factor & pure linear stress state \\
\hline$l$ & length & M matrix failure path \\
\hline$m$ & Weibull shape parameter & mean value \\
\hline$n$ & number of elements / fibres & $\mathrm{n} \quad$ chain of elements \\
\hline$s$ & interfibre spacing & $r \quad$ reference length / element \\
\hline$\sigma$ & longitudinal stress & SL shear-lag \\
\hline$\tau$ & shear (yield) stress & U uniform stress state \\
\hline
\end{tabular}

Several theories have been proposed to model the relation between size and strength of structures. The stochastic approach, based on the Weakest Link Theory (WLT) and formalised by Weibull (1951), has been extensively applied to FRPs (Bažant and Pang, 2007, Wisnom, 1999). Deterministic size effects have also been studied by Bažant (1999, due to the energy dissipated by failure of quasi-brittle materials), Carpinteri (1994, due to the fractal self-similar failure of heterogeneous materials), and Wisnom et al. (2010, due to the change of failure mode in scaled laminated composites). All authors agree nevertheless that stochastic size effects are key for the strength of FRPs.

The WLT states that a chain withstands an external load only if all its elements survive the resulting stresses. Let $X_{\mathrm{U}, \mathrm{r}}$ be the stochastic strength of the elements (with reference length $l_{\mathrm{r}}$, all statistically identical and independent) under an uniform (subscript $\mathrm{U})$ stress $\sigma$; the survival probability for each element is $S_{\mathrm{U}, \mathrm{r}}(\sigma)=\operatorname{Pr}\left(X_{\mathrm{U}, \mathrm{r}}>\sigma\right)$. Therefore, the survival probability of a chain (length $l_{\mathrm{n}}=n \cdot l_{\mathrm{r}}$ ) with $n$ elements is:

$$
S_{\mathrm{U}, \mathrm{n}}(\sigma)=\left[S_{\mathrm{U}, \mathrm{r}}(\sigma)\right]^{n} \quad \Rightarrow \quad \ln \left[S_{\mathrm{U}, \mathrm{n}}(\sigma)\right]=n \cdot \ln \left[S_{\mathrm{U}, \mathrm{r}}(\sigma)\right]=\frac{l_{\mathrm{n}}}{l_{\mathrm{r}}} \cdot \ln \left[S_{\mathrm{U}, \mathrm{r}}(\sigma)\right]
$$

Weibull (1951) proposed a distribution for the strength of brittle materials, so that survival and failure probabilities of a chain under uniform stresses $\sigma$ are:

$$
S_{\mathrm{U}, \mathrm{n}}(\sigma)=\exp \left[-\frac{l_{\mathrm{n}}}{l_{\mathrm{r}}}\left(\frac{\sigma}{\sigma_{0}}\right)^{m}\right] \quad \text { and } \quad F_{\mathrm{U}, \mathrm{n}}(\sigma)=1-\exp \left[-\frac{l_{\mathrm{n}}}{l_{\mathrm{r}}}\left(\frac{\sigma}{\sigma_{0}}\right)^{m}\right]
$$


where $m$ and $\sigma_{0}$ are respectively the shape (size independent) and scale (measured at $l_{\mathrm{r}}$ ) parameters of the distribution.

Equation 2 has been widely used to model the length effect on the strength of technical fibres, e.g. glass or carbon (Curtin, 1999, Pradhan et al., 2010, Wisnom, 1999). While alternative strength distributions have been proposed (e.g. Beetz, 1982, Beyerlein and Phoenix, 1996a), Stoner et al. (1994) showed that a good agreement between Weibull's theory and experimental measurements can be achieved by taking the spurious effect of fibre gripping and testing variability into account.

At the FRP level, the parallel fibre arrangement and presence of matrix result in a quasi-brittle failure. Beyerlein and Phoenix (1996a) and Kazanci (2004) tested microbundles (with 4 and 7 fibres respectively) and found that bundle strengths deviated significantly from Weibull distributions; moreover, some bundles (depending on the resin) had higher mean strength than the single-fibres, but considerably lower variability. At the macroscopic scale, Okabe and Takeda (2002) and Scott et al. (2011) observed several clusters of fibre breaks before final coupon failure. Wisnom (1999) also noted that both the magnitude of size effects and the variability of strength decrease for larger specimens. All these observations are incompatible with the WLT applied directly to the single-fibre level (Bažant, 1999, Wisnom, 1999).

The asymptotic behaviour of tensile strength of FRPs has been successfully modelled in the literature (see reviews from Bažant, 1999, Curtin, 1999, Wisnom, 1999). However, the recent developments in composites with thin plies (Sihn et al., 2007) and with discontinuous reinforcement - with individual fibres and bundles of various sizes (Harper et al., 2007, Pimenta et al., 2010) — requires developing and validating full scaling models. Wisnom (1999) suggests Fibre Bundle Models (FBMs, firstly developed by Daniels, 1945, and recently reviewed by Pradhan et al., 2010) have the potential to capture most of the physics involved in longitudinal tensile failure of FRPs and the associated size effects.

Several FBMs have been proposed to predict the longitudinal tensile strength and size effects in composites. The key challenge is to calculate the strength distribution of a bundle of parallel fibres with a given characteristic length, to then use the WLT (Equation 1) to scale the result for a chain of bundles (Curtin, 1991, Harlow and Phoenix, 1978a,b). Different fibre arrangements, matrix responses, load sharing schemes and magnitudes of stress concentrations have been assumed (see review from Curtin, 1999).

The complexity of most FBMs increases exponentially with the number of fibres considered, hence exact solutions are attainable for small bundles only; consequently, 
much work focused on asymptotic analyses (Curtin, 1993, Phoenix et al., 1997, Phoenix and Smith, 1983) and Monte-Carlo simulations (Landis et al., 2000, Mahesh et al., 2002, Okabe and Takeda, 2001, Wada and Fukuda, 1999). Most authors use a characteristic length independent of load and number of fibre breaks, although this is known not to be correct (Behzadi et al., 2009, Phoenix et al., 1988).

Laffan et al. (2010) and Pimenta et al. (2010) reported self-similar or quasi-fractal fracture surfaces in thin (under $0.5 \mathrm{~mm}$ ) UD laminas and fibre bundles; this provides experimental evidence that the length-scale of the failure process increases with the number of fibres involved. Moreover, such observations suggest a hierarchical failure process, hence supporting the use of hierarchical models - e.g. Newman and Gabrielov's (1991) model for $d r y$ bundles. Here, considering that a bundle of level $[i+1]$ is composed by two sub-bundles of level $[i]$, strength distributions were calculated recursively as:

$$
F^{[i+1]}(\sigma)=F^{[i]}(\sigma) \cdot\left[2 \cdot F^{[i]}(2 \cdot \sigma)-F^{[i]}(\sigma)\right]
$$

where $F^{[i]}(\sigma)$ is the failure probability of a level-[i] bundle under an applied stress $\sigma$. The recursive nature of this scaling law also allowed its efficient implementation, so that large-scale bundles could be computed. However - being a model for dry bundles - it does not consider the effect of an embedding matrix, and does not include any characteristic length (which is paramount for quasi-brittle materials, Bažant, 1999); the model is also inconsistent with the WLT for length scaling.

Altogether, a comprehensive explanation of the micromechanics and statistics of tensile failure in composites is yet to be provided, as are validated quantitative predictions over a complete range of scales. Still, FBMs surface as one of the most promising approaches to overcome this knowledge gap.

This paper presents the development, implementation and validation of a FBM for predicting size effects on the longitudinal tensile strength of composite bundles. Following Newman and Gabrielov's (1991) work, bundles are hierarchically organised; however, the role of the matrix (or fibre-matrix interface) is now considered through a simplified shear-lag model, in which the characteristic length scales hierarchically as well.

This paper is organised as follows: Section 2 presents the analytical model for predicting strength distributions of FRP bundles of different dimensions. Section 3 explores modelling results (including experimental validation), subsequently discussed in Section 4. Finally, Section 5 draws the main conclusions. 


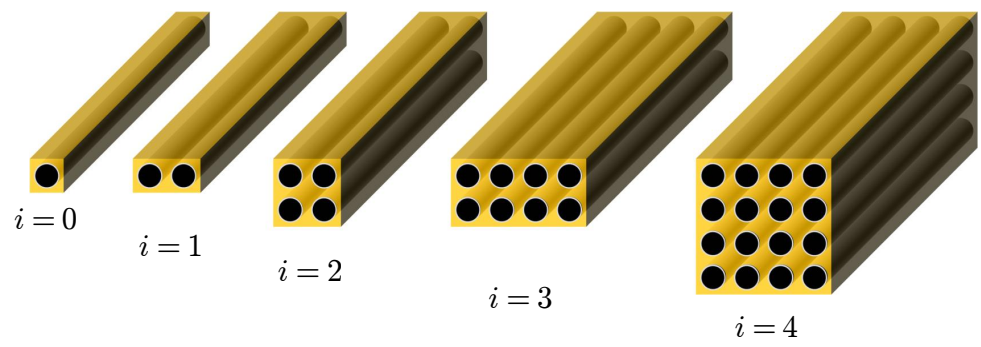

(a) Bundle hierarchy.

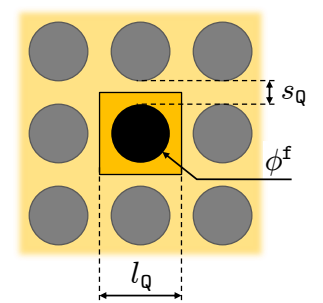

(b) Fibre arrangement.

Figure 1: Hierarchical bundles in square fibre arrangement.

\section{Model development}

\subsection{Fibre bundle geometry and shear-lag boundary}

This model is based on hierarchical fibre-matrix bundles (Figure 1a). These are generated by pairing individual fibres (level-[0]) into level-[1] bundles, and then sequentially grouping two level-[i] bundles into one level- $[i+1]$ bundle (Newman and Gabrielov, 1991). The number of fibres $\left(n^{[i]}\right)$ in a level- $[i]$ bundle is therefore:

$$
n^{[i]}=2^{i} \quad \Leftrightarrow \quad i=\log _{2} n^{[i]}
$$

The fibres (superscript $\mathrm{f}$, diameter $\phi^{\mathrm{f}}$, circumference $C^{\mathrm{f}}$ and area $A^{\mathrm{f}}$ ) are embedded in the matrix (with volume fraction $V^{\mathrm{f}}$ ) in a square architecture (Figure 1b).

During hierarchical failure of a large composite bundle (Figure 2a), shear-lag stresses will be transferred between the (unbroken) surrounding material and a broken level- $[i]$ bundle through the shear-lag boundary, with perimeter $C^{[i]}$. Considering preferential interfacial debonding (Figure 2b),

$$
C^{[i]}=3 \cdot C^{\mathrm{f}}+4 \cdot\left[\left(\sqrt{n^{[i]}}-1\right) \cdot s_{\mathrm{Q}}+\left(\sqrt{n^{[i]}}-2\right) \cdot \frac{C^{\mathrm{f}}}{2}\right], \text { with } \quad s_{\mathrm{Q}}=\left(\frac{\sqrt{\pi}}{2 \cdot \sqrt{V^{\mathrm{f}}}}-1\right) \cdot \phi^{\mathrm{f}}
$$

This expression is strictly valid only for even values of $i$, but used for any bundle size so that $C^{[i]}$ is a smooth function of $n^{[i]}$.

Other geometries for bundles and their boundaries (e.g. hexagonal fibre arrangement with fractal boundary, preferential matrix failure, free-edge effects) are considered in Appendix A. These variations are shown to have a minor influence on calculated bundle strength distributions (as already suggested by Curtin and Takeda, 1998). 

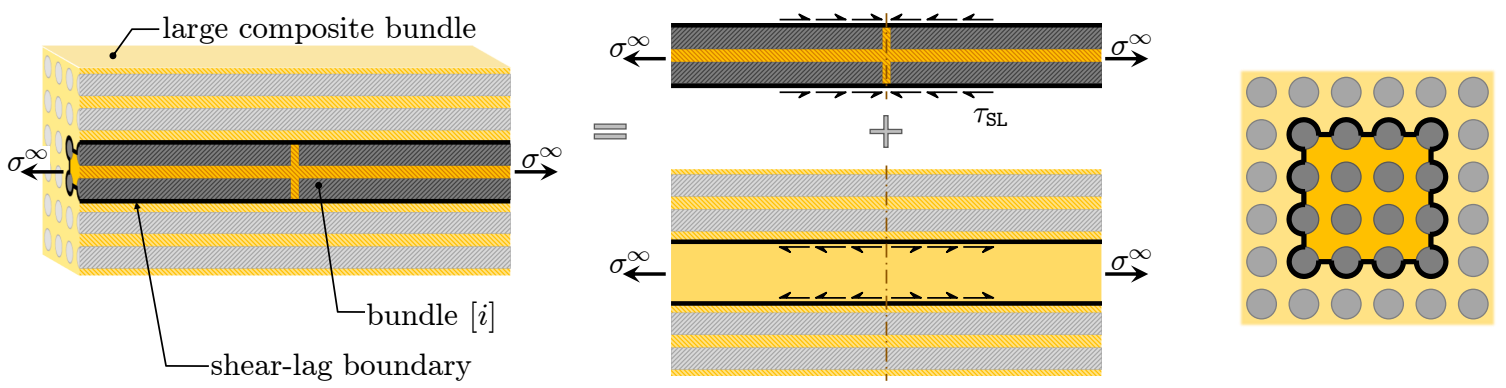

(a) Longitudinal view.

(b) Section view.

Figure 2: Shear-lag boundary (assuming preferential interfacial failure).

\subsection{Stress field around a fibre break and definition of the control region}

Consider a level-[1] bundle of reference length $l_{\mathrm{r}}$, composed by two level-[0] fibres $(\mathcal{A}$ and $\mathcal{B})$ in a soft matrix $(i=1$ in Figure 1a). The bundle is loaded in tension by a progressively increasing remote stress $\sigma^{\infty}$, so that each fibre undergoes a uniform stress state $\sigma^{\mathcal{A}}(x)=\sigma^{\mathcal{B}}(x)=\sigma^{\infty}$. Note that longitudinal stresses are expressed as fibre stresses, i.e. normalised by the area of fibres in the cross section.

Assume that fibre $\mathcal{A}$ fails at the location $x=0$ under a given $\sigma^{\infty}$ (Figure 3a). Shear-lag models have been shown to accurately reproduce the resulting stress fields, as validated by more complex Finite Element analyses (Landis and McMeeking, 1999, de Morais, 2001). The in-situ response of the matrix / interface to this event is complex, as for instance epoxy is usually brittle in bulk, but actually ductile and much stronger in-situ (Gulino et al., 1991, Hobbiebrunken et al., 2007, de Morais, 2001). This, together with the lack of agreement in the literature on whether fibre failure should be modelled through energy or stress based approaches (Nairn, 1997, Zhandarov et al., 1998), supports the use of a perfectly-plastic shear-lag approach, for the sake of simplicity (Landis and McMeeking, 1999, de Morais, 2006).

According to perfectly-plastic shear-lag, the failed fibre $\mathcal{A}$ recovers the remote stress $\sigma^{\infty}$ within the level-[0] effective recovery length (subscript e), defined as (Kelly and Tyson, 1965):

$$
l_{\mathrm{e}}^{[0]}\left(\sigma^{\infty}\right)=2 \cdot \frac{A^{\mathrm{f}}}{C^{[0]} \cdot \tau_{\mathrm{SL}}} \cdot \sigma^{\infty}
$$

Here, $\tau_{\mathrm{SL}}$ is the matrix / interface yield stress, and $C^{[0]}$ is the perimeter of the level- $[0]$ shear-lag boundary (Equation 5 implies $C^{[0]}=C^{\mathbf{f}}$ ).

Conversely, fibre $\mathcal{B}$ undergoes linear stress concentrations within the length $l_{\mathrm{e}}^{[0]}$ (Figure $3 \mathrm{a}$ ). In this model, equilibrium of the 2 fibres yields a stress concentration factor 


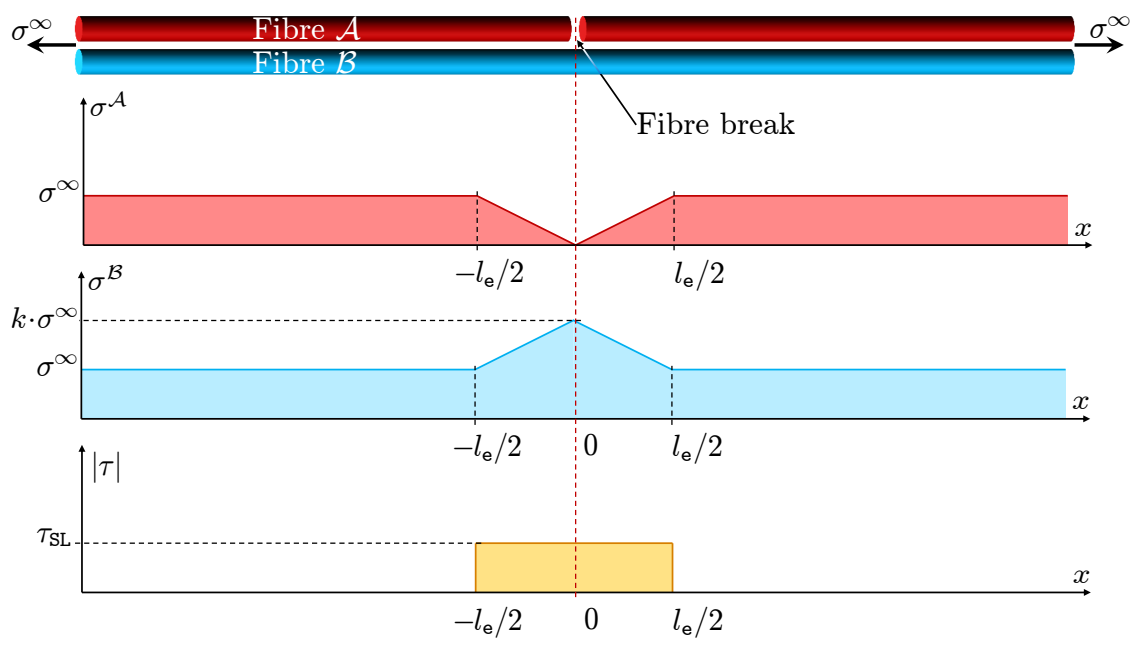

(a) Stress fields after first fibre failure.

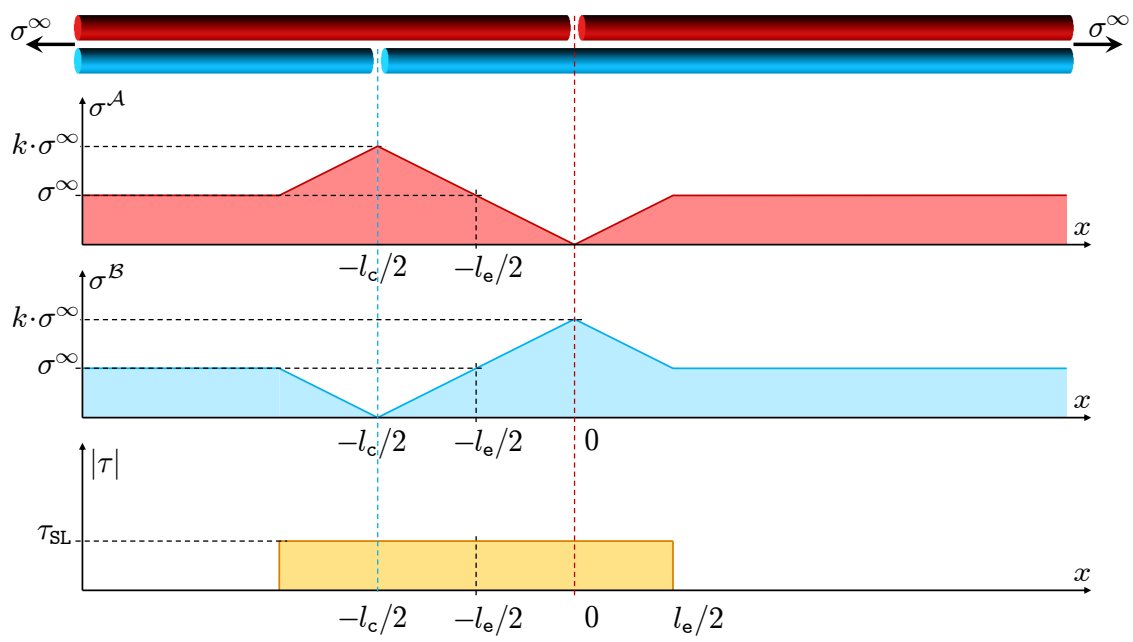

(b) Definition of critical distance between fibre breaks: the bundle fails only if fibre $\mathcal{B}$ breaks at a distance smaller than $l_{\mathrm{c}} / 2$ from the break in fibre $\mathcal{A}$.

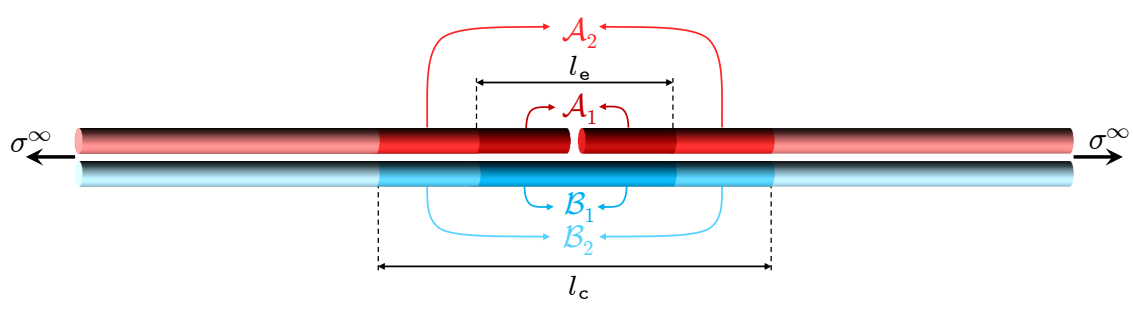

(c) Definition of the control region and fibre segments.

Figure 3: Stress fields and length scales in a level-[1] fibre bundle. 
$k=2$. However, the true value of $k$ near a cluster of fibre breaks is still an open issue, and depends on local fibre arrangement, size of broken cluster, matrix response and dynamic effects (Accorsi et al., 1996, Beyerlein and Phoenix, 1996b, Hedgepeth, 1961, Mahesh et al., 2002, de Morais, 2001, Nedele and Wisnom, 1994, Swolfs et al., 2013). Therefore, $k$ will be treated as free parameter.

Bundle failure requires that both fibres $\mathcal{A}$ and $\mathcal{B}$ break in nearby locations, so as to promote complete yielding of matrix / interface between breaks (Figure 3b). Therefore, once fibre $\mathcal{A}$ fails, the level-[1] control region (subscript c) — within which a break in fibre $\mathcal{B}$ leads to bundle failure — has a length defined by:

$$
l_{\mathrm{c}}^{[1]}\left(\sigma^{\infty}\right)=2 \cdot l_{\mathrm{e}}^{[0]}\left(\sigma^{\infty}\right)=4 \cdot \frac{A^{\mathrm{f}}}{C^{[0]} \cdot \tau_{\mathrm{SL}}} \cdot \sigma^{\infty}
$$

This region, centred at the first fibre break, is partitioned into 4 fibre segments $\left(\mathcal{A}_{1}\right.$, $\mathcal{A}_{2}, \mathcal{B}_{1}$ and $\mathcal{B}_{2}$ ) of equal length $l_{\mathrm{e}}^{[0]}$ (Figure 3c). The probability of failure of the level-[1] bundle within the control length will be determined in Section 2.3.

This definition of $l_{\mathrm{c}}^{[1]}$ differs from other characteristic lengths in the literature. Firstly, $l_{\mathrm{c}}^{[1]} \propto \sigma^{\infty}$ (as opposed to fixing $l_{\mathrm{c}}^{[1]}$ at a characteristic stress, as done by Curtis, 1986, Fukuda and Kawata, 1977, Harlow and Phoenix, 1978a, Okabe and Takeda, 2001). Secondly, $l_{\mathrm{c}}^{[1]}=2 \cdot l_{\mathrm{e}}^{[0]}$; while Curtin (1993), Phoenix et al. (1997) proposed $l_{\mathrm{c}}^{[1]} \leq l_{\mathrm{e}}^{[0]}$ for global load sharing, Equation 7 defines the shortest statistically independent partition of a level-[1] bundle. Finally, the control length of a larger level-[i+1] bundle will be scaled hierarchically in Section 2.4.

\subsection{Statistical analysis of level-[1] bundle failure}

The strength distribution of the level-[1] bundle analysed in Figure 3 will be determined under the following assumptions:

A(i) At each remote stress $\sigma^{\infty}$, the bundle is represented by a chain of independent control regions of length $l_{\mathrm{c}}^{[1]}$ (Equation 7 ). This shifts the first fibre break to the centre, thus neglecting bundle--end effects.

A(ii) Within a control region, each fibre may break only once (equivalent to the WLT); this guarantees that the stress fields presented in Figure 3 are always valid.

Statistically, a level-[1] bundle survives the remote stress $\sigma^{\infty}$ in the control length if (i) all its 4 fibre segments survive the uniform stress $\sigma^{\infty}$, or (ii) the weakest fibre 
fails under $\sigma^{\infty}$ and the strongest fibre survives the resulting stress field - comprising one segment under the uniform stress $\sigma^{\infty}$, and another segment under linear stress concentrations (factor $k$ ). The survival probability of the level-[1] bundle under uniform (subscript U) stresses $\sigma^{\infty}$ within the control length is then:

$$
S_{\mathrm{U}, \mathbf{c}}^{[1]}\left(\sigma^{\infty}\right)=S_{\mathrm{U}, \mathbf{e}}^{[0]}\left(\sigma^{\infty}\right)^{4}+2 \cdot\left[1-S_{\mathrm{U}, \mathbf{e}}^{[0]}\left(\sigma^{\infty}\right)^{2}\right] \cdot S_{\mathrm{U}, \mathbf{e}}^{[0]}\left(\sigma^{\infty}\right) \cdot S_{\mathrm{K}, \mathbf{e}}^{[0]}\left(\sigma^{\infty}\right) \quad, \quad \text { where: }
$$

- $S_{\mathrm{U}, \mathbf{e}}^{[0]}\left(\sigma^{\infty}\right)$ is the survival probability of a single-fibre segment loaded by a uniform stress $\sigma^{\infty}$ within the length $l_{\mathrm{e}}^{[0]}$. Assuming that fibre strength follows a Weibull distribution with survival probability $S_{\mathrm{U}, \mathrm{r}}^{[0]}$ at the reference length $l_{\mathrm{r}}$ (with parameters $m$ and $\sigma_{0}^{\mathrm{f}}$ as shown below), then WLT scaling (Equation 2) implies:

$$
S_{\mathrm{U}, \mathrm{r}}^{[0]}=\exp \left[-\left(\frac{\sigma^{\infty}}{\sigma_{0}^{\mathrm{f}}}\right)^{m}\right] \quad \text { and } \quad S_{\mathrm{U}, \mathrm{e}}^{[0]}=\exp \left[-\frac{l_{\mathrm{e}}^{[0]}}{l_{\mathrm{r}}} \cdot\left(\frac{\sigma^{\infty}}{\sigma_{0}^{\mathrm{f}}}\right)^{m}\right]
$$

- $S_{\mathrm{K}, \mathbf{e}}^{[0]}\left(\sigma^{\infty}\right)$ is the survival probability of a single-fibre segment loaded under linear stress concentrations (variable remote stress $\sigma^{\infty}$ and constant factor $k$ ) within the length $l_{\mathrm{e}}^{[0]}$. Generalising the WLT to non-uniform chain stresses (Appendix B),

$$
S_{\mathrm{K}, \mathrm{e}}^{[0]}=\exp \left[-\mathcal{C}_{\mathrm{K}} \cdot \frac{l_{\mathrm{e}}^{[0]}}{l_{\mathrm{r}}} \cdot\left(\frac{\sigma^{\infty}}{\sigma_{0}^{\mathrm{f}}}\right)^{m}\right] \quad \text { where } \quad \mathcal{C}_{\mathrm{K}}=\frac{k^{m+1}-1}{(m+1) \cdot(k-1)}
$$

While the level-[1] scaling law in Equation 8 was derived from a purely statistical argument, Appendix $\mathrm{C}$ shows it actually corresponds to the following physical sequences of events leading to bundle failure:

$E_{1}$ : failure of the weakest fibre and immediate (unstable) failure (with no increment of $\sigma^{\infty}$ ) of segment $\mathcal{B}_{1}$ due to stress concentrations;

$E_{2}$ : failure of the weakest fibre and later (after incrementing $\sigma^{\infty}$ ) failure of the stress concentrations segment $\mathcal{B}_{1}$;

$E_{3}$ : failure of the weakest fibre and later failure of the far-segment $\mathcal{B}_{2}$ (stable failure due to independent fibre flaws). This includes bundle failure by growth and coalescence of matrix damage between two previously formed breaks. 


\subsection{Hierarchical law for bundle failure}

Equation 8 relates the strength distribution of a single fibre to that of a level-[1] bundle. Assuming a self-similar hierarchical failure process as reported by Laffan et al. (2010), Pimenta et al. (2010), this can be extrapolated to any bundle level and used recursively throughout bundle hierarchy. Consequently, the survival probability of a level- $[i+1]$ bundle under uniform stresses is (omitting $\sigma^{\infty}$ for readability):

$$
S_{\mathrm{U}, \mathrm{c}}^{[i+1]}=\left(S_{\mathrm{U}, \mathrm{e}}^{[i]}\right)^{4}+2 \cdot\left[1-\left(S_{\mathrm{U}, \mathrm{e}}^{[i]}\right)^{2}\right] \cdot S_{\mathrm{U}, \mathrm{e}}^{[i]} \cdot S_{\mathrm{K}, \mathrm{e}}^{[i]}
$$

Level- $[i]$ survival probabilities on the right-hand side are defined at the respective effective recovery length $l_{\mathrm{e}}^{[i]}\left(\sigma^{\infty}\right)$, while $S_{\mathrm{U}, \mathrm{c}}^{[i+1]}$ is defined at the level- $[i+1]$ control length. Contrarily to most fibre bundle models in the literature (Curtin, 1991, Curtis, 1986, Fukuda and Kawata, 1977, Harlow and Phoenix, 1978a, Okabe and Takeda, 2001), these characteristic lengths are also scaled hierarchically from their original definition. Using the shear-lag perimeters defined in Equation 5:

$$
l_{\mathrm{e}}^{[i]}\left(\sigma^{\infty}\right) \stackrel{\text { Eq. }}{=} 6 \cdot \frac{n^{[i]} \cdot A^{\mathrm{f}}}{C^{[i]} \cdot \tau_{\mathrm{SL}}} \cdot \sigma^{\infty} \quad, \quad l_{\mathrm{c}}^{[i+1]}\left(\sigma^{\infty}\right) \stackrel{\text { Eq. }}{=} 7 \cdot l_{\mathrm{e}}^{[i]}\left(\sigma^{\infty}\right)
$$

Equation 11 can be written in both the following logarithmic forms:

$$
\begin{aligned}
\ln \left(S_{\mathrm{U}, \mathrm{e}}^{[i+1]}\right) & =4 \cdot \ln \left(S_{\mathrm{U}, \mathrm{e}}^{[i]}\right)+\ln \left(1+2 \cdot \frac{S_{\mathrm{K}, \mathrm{e}}^{[i]}}{\left(S_{\mathrm{U}, \mathrm{e}}^{[i]}\right)^{3}}-2 \cdot \frac{S_{\mathrm{K}, \mathrm{e}}^{[i]}}{S_{\mathrm{U}, \mathrm{e}}^{[i]}}\right)= \\
& =\ln \left(S_{\mathrm{U}, \mathrm{e}}^{[i]}\right)+\ln \left(S_{\mathrm{K}, \mathrm{e}}^{[i]}\right)+\ln \left(2+\frac{\left(S_{\mathrm{U}, \mathrm{e}}^{[i]}\right)^{3}}{S_{\mathrm{K}, \mathrm{e}}^{[i]}}-2 \cdot\left(S_{\mathrm{U}, \mathrm{e}}^{[i]}\right)^{2}\right)
\end{aligned}
$$

Scaling to the reference length (using the WLT in Equation 1 and $l_{\mathrm{c}}^{[i+1]}$ in Equation 12),

$$
\begin{aligned}
\ln \left(S_{\mathrm{U}, \mathrm{r}}^{[i+1]}\right) & =2 \cdot \ln \left(S_{\mathrm{U}, \mathrm{r}}^{[i]}\right)+\frac{l_{\mathrm{r}}}{2 \cdot l_{\mathrm{e}}^{[i]}} \cdot \ln \left(1+2 \cdot\left[\frac{S_{\mathrm{K}, \mathrm{r}}^{[i]}}{\left(S_{\mathrm{U}, \mathrm{r}}^{[i]}\right)^{3}}\right]^{l_{\mathrm{e}}^{[i]} / l_{\mathrm{r}}}-2\left[\cdot \frac{S_{\mathrm{K}, \mathrm{r}}^{[i]}}{S_{\mathrm{U}, \mathrm{r}}^{[i]}}\right]^{l_{\mathrm{e}}^{[i]} / l_{\mathrm{r}}}\right)= \\
& =\frac{\ln \left(S_{\mathrm{U}, \mathrm{r}}^{[i]}\right)+\ln \left(S_{\mathrm{K}, \mathrm{r}}^{[i]}\right)}{2}+\frac{l_{\mathrm{r}}}{2 \cdot l_{\mathrm{e}}^{[i]}} \cdot \ln \left(2+\left[\frac{\left(S_{\mathrm{U}, \mathrm{r}}^{[i]}\right)^{3}}{S_{\mathrm{K}, \mathrm{r}}^{[i]}}\right]^{l_{\mathrm{e}}^{[i]} / l_{\mathrm{r}}}-2 \cdot\left[\left(S_{\mathrm{U}, \mathrm{r}}^{[i]}\right)^{2}\right]^{l_{\mathrm{e}}^{[i]} / l_{\mathrm{r}}}\right)
\end{aligned}
$$

The expressions above are analytically equivalent but prone to different numerical errors (due to exponential arguments); consequently, both forms will be used (Section 2.6). 
The level-[i] survival probability under linear stress concentrations $S_{\mathrm{K}, \mathrm{r}}^{[i]}\left(\sigma^{\infty}\right)$ can be calculated from $S_{\mathrm{U}, \mathrm{r}}^{[i]}\left(\sigma^{\infty}\right)$. Bundle strengths do not follow Weibull distributions when $i>0$, hence the generic relation derived in Appendix B must be employed:

$$
\begin{aligned}
& \ln \left[S_{\mathrm{K}, \mathrm{r}}^{[i]}\left(\sigma^{\infty}\right)\right]= \begin{aligned}
\frac{k \cdot \ln \left[S_{\mathrm{L}, \mathrm{r}}^{[i]}\left(k \cdot \sigma^{\infty}\right)\right]-\ln \left[S_{\mathrm{L}, \mathrm{r}}^{[i]}\left(\sigma^{\infty}\right)\right]}{k-1} & \text { if } \quad k>1 \\
\ln \left[S_{\mathrm{U}, \mathrm{r}}^{[i]}\left(\sigma^{\infty}\right)\right] & \text { if } \quad k=1
\end{aligned} \\
& \text { where } \quad \ln \left[S_{\mathrm{L}, \mathrm{r}}^{[i]}(\sigma)\right]=\frac{1}{\sigma} \int_{\sigma_{\mathrm{L}=0}}^{\sigma} \ln \left[S_{\mathrm{U}, \mathrm{r}}^{[i]}\left(\sigma_{\mathrm{L}}\right)\right] \mathrm{d} \sigma_{\mathrm{L}}
\end{aligned}
$$

\subsection{Asymptotic behaviour}

If fibre strength follows a Weibull distribution (Equation 9), then each tail of any bundle strength distributions tends asymptotically to a Weibull as well (Appendix D):

- The Right Tail Asymptote (RTA, $\sigma^{\infty} \rightarrow \infty$ ) of a bundle survival probability is:

$$
\begin{aligned}
& S_{\mathrm{U}, \mathrm{r}, \mathrm{RTA}}^{[i]}\left(\sigma^{\infty}\right)=\exp \left[-\left(\frac{\sigma^{\infty}}{\sigma_{0, \mathrm{RTA}}^{[i]}}\right)^{m}\right], \text { with: } \\
& \sigma_{0, \mathrm{RTA}}^{[i]}= \begin{cases}2^{-i / m} \cdot \sigma_{0}^{\mathrm{f}} & \text { if } \quad \mathcal{C}_{\mathrm{K}} \geq 3 \\
\left(\frac{1+\mathcal{C}_{\mathrm{K}}}{2}\right)^{-i / m} \cdot \sigma_{0}^{\mathrm{f}} & \text { if } \quad \mathcal{C}_{\mathrm{K}}<3\end{cases}
\end{aligned}
$$

This preserves the single-fibre shape parameter $m$. As most technical fibres (e.g. carbon and glass) have $\mathcal{C}_{\mathrm{K}} \geq 3$ ( $m \gtrsim 2.6$ with $k=2$ in Equation 10), the RTA of a bundle strength distribution corresponds to the WLT applied to the fibre level. This has been observed in other FBMs as well (Harlow and Phoenix, 1978b).

- The Left Tail Asymptote (LTA, $\sigma^{\infty} \rightarrow 0$ ) of a bundle survival probability is defined recursively as:

$$
S_{\mathrm{U}, \mathrm{r}, \mathrm{LTA}}^{[i+1]}\left(\sigma^{\infty}\right)=\exp \left[-4 \cdot \mathcal{C}_{\mathrm{K}, \mathrm{LTA}}^{[i]} \cdot \frac{n^{[i]} \cdot A^{\mathrm{f}} \cdot \sigma_{0, \mathrm{LTA}}^{[i]}}{C^{[i]} \cdot \tau_{\mathrm{SL}} \cdot l_{\mathrm{r}}} \cdot\left(\frac{\sigma^{\infty}}{\sigma_{0, \mathrm{LTA}}^{[i]}}\right)^{2 \cdot m_{\mathrm{LTA}}^{[i]}+1}\right]
$$

Level- $[i]$ parameters are defined in Equation D.8. It should be noticed that the shape parameter of LTAs more than doubles with each hierarchical level; therefore, one can expect lower variability for the strength of larger bundles. Such behaviour 
is typical of FBMs (Harlow and Phoenix, 1978b), although $m_{\text {LTA }}$ here increases in a more pronounced way (due to $l_{\mathrm{c}}^{[i+1]} \propto \sigma^{\infty}$ ).

In addition to providing further insight on bundle strength distributions and size effects, the RTA behaviour is key for the implementation of this model (Section 2.6). Equations 14 and 15 show that defining $S_{\mathrm{U}, \mathrm{r}}^{[i+1]}\left(\sigma^{\infty}\right)$ requires calculating $S_{\mathrm{U}, \mathrm{r}}^{[i]}\left(k \cdot \sigma^{\infty}\right)$, and thus (following a recursive procedure down to the single-fibre level) calculating $S_{\mathrm{U}, \mathrm{r}}^{[0]}\left(k^{i+1} \cdot \sigma^{\infty}\right)$. This becomes intractable as bundle level increases. Fortunately, the Weibull-like behaviour of RTAs (Equation 16) allows replacing the general relation between $S_{\mathrm{U}, \mathrm{r}}^{[i]}\left(\sigma^{\infty}\right)$ and $S_{\mathrm{K}, \mathrm{r}}^{[i]}\left(\sigma^{\infty}\right)$ (Equation 15) with its closed-form result for Weibull distributions (Equation B.7), when $\sigma^{\infty} \rightarrow \infty$ :

$$
\lim _{\sigma^{\infty} \rightarrow \infty} \ln \left[S_{\mathrm{K}, \mathrm{r}}^{[i]}\left(\sigma^{\infty}\right)\right]=\mathcal{C}_{\mathrm{K}} \cdot \ln \left[S_{\mathrm{U}, \mathrm{r}}^{[i]}\left(\sigma^{\infty}\right)\right] \quad, \forall i \geq 0
$$

This eliminates the need to compute $S_{\mathrm{U}, \mathrm{r}}^{[i]}\left(k \cdot \sigma^{\infty}\right)$ for large values of $\sigma^{\infty}$.

\subsection{Numerical implementation}

An overview of the numerical implementation of the present model is shown in Figure 4; using array programming (e.g. MATLAB) greatly simplifies the implementation and reduces running time, hence the following remarks are of relevance:

- In the numerical implementation, the remote stress $\sigma^{\infty}$ becomes a discrete vector $\boldsymbol{\sigma}$, with $n_{\boldsymbol{\sigma}}$ evenly spaced stress values from $\sigma^{\infty}=0$ to $\sigma^{\infty} \geq \sigma^{\max }$ (step size $\Delta \sigma$ );

- Accordingly, most variables are expressed in vectorial form (indicated in upright bold in Figure 4); all arithmetic operators represent pointwise calculations. Survival distributions are stored in logarithmic form, $\ln S \equiv\left\{\ln \left[S\left(\sigma^{\infty}\right)\right]\right\}$;

- Integration symbols represent numerical integration; these can be efficiently evaluated using MATLAB's in-built trapz and cumtrapz functions;

- Module IV calculates bundle strength distributions $F_{\mathrm{U}, \mathrm{r}}^{[i]}(\sigma)=\operatorname{Pr}\left(X_{\mathrm{U}, \mathrm{r}}^{[i]} \leq \sigma\right)$, where $X_{\mathrm{U}, \mathrm{r}}^{[i]}$ is the stochastic strength of a level-[i] bundle of length $l_{\mathrm{r}}$ under uniform stresses. Mean values $\left(X_{\mathrm{m}}^{[i]}\right)$ and Coefficients of Variation $\left(\mathrm{CoV}_{X}^{[i]}\right)$ depend directly on the cumulative distribution function, as a result of integration by parts. 


\section{Definition of input variables}

I.1: Numerical variables $\Delta \sigma, \sigma^{\max }$
I.2: Single fibre strength

$l_{\mathrm{r}}, \sigma_{0}^{\mathrm{f}}, m$
I.3: Composite bundle $\tau_{\mathrm{SL}}, V^{\mathrm{f}}, \phi^{\mathrm{f}}, k, i^{\max }$

\section{Preliminary calculations}

II.1: Define strength vector
$n_{\boldsymbol{\sigma}}=\left\lceil\frac{\sigma^{\max }}{\Delta \sigma}\right\rceil+1$
$\boldsymbol{\sigma}=\{(j-1) \cdot \Delta \sigma\}_{j=1}^{n_{\boldsymbol{\sigma}}}$
$n_{\mathrm{K}}=\left\lfloor\frac{n_{\boldsymbol{\sigma}}-1}{k}\right\rfloor+1$

II.2: Define geometric parameters
$A^{\mathrm{f}}=\pi \cdot \frac{\left(\phi^{\mathrm{f}}\right)^{2}}{4}$
$C^{\mathrm{f}}=\pi \cdot \phi^{\mathrm{f}}$
$s_{\mathrm{SL}}=\left(\frac{\sqrt{\pi}}{2 \cdot \sqrt{V^{\mathrm{f}}}}-1\right) \cdot \phi^{\mathrm{f}}$

II.3: Calculate single-fibre log-survival vectors
$\ln \mathbf{S}_{\mathbf{U r}}^{[0]}=-\left(\frac{\boldsymbol{\sigma}}{\sigma_{0}^{f}}\right)^{m}$
$\mathcal{C}_{\mathrm{K}}=\frac{k^{m+1}-1}{(k-1) \cdot(m+1)}$
$\operatorname{lnS}_{\mathbf{K r}}^{[0]}=-\mathcal{C}_{\mathrm{K}}\left(\frac{\boldsymbol{\sigma}}{\sigma_{0}^{\mathrm{f}}}\right)^{m}$

\section{Strength scaling model}

III.0: Set bundle level counter $i=0$

$\longrightarrow i=i+1$

III.1: Define geometry and normalised effective length vector:

$$
n^{[i-1]}=2^{i-1} \quad C^{[i-1]}=3 \cdot C^{\mathrm{f}}+4 \cdot\left[\left(\sqrt{n^{[i-1]}}-1\right) \cdot s_{\mathrm{SL}}+\left(\sqrt{n^{[i-1]}}-2\right) \cdot C^{\mathrm{f}} / 2\right] \quad \mathbf{l}_{\mathbf{e} / \mathbf{r}}^{[i-1]}=2 \cdot \frac{n^{[i-1]} \cdot A^{\mathrm{f}}}{C^{[i-1]} \cdot \tau_{\mathrm{SL}} \cdot l_{\mathrm{r}}} \cdot \boldsymbol{\sigma}
$$

III.2: Calculate uniform-stresses bundle survival vector

$$
\begin{aligned}
& \operatorname{lnS}_{\mathbf{U r}}^{[i]}=2 \cdot \ln \mathbf{S}_{\mathbf{U r}}^{[i-1]}+\frac{1}{2 \cdot \mathbf{l}_{\mathbf{e} / \mathbf{r}}^{i-1}} \cdot \ln \left[1+2 \cdot \exp \left(\mathbf{l}_{\mathbf{e} / \mathbf{r}}^{[i-1]} \cdot\left[\ln \mathbf{S}_{\mathbf{K r}}^{[i-1]}-3 \cdot \ln \mathbf{S}_{\mathbf{U r}}^{[i-1]}\right]\right)-2 \cdot \exp \left(\mathbf{l}_{\mathbf{e} / \mathbf{r}}^{[i-1]} \cdot\left[\ln \mathbf{S}_{\mathbf{K r}}^{[i-1]}-\ln \mathbf{S}_{\mathbf{U r}}^{[i-1]}\right\rceil\right)\right]
\end{aligned}
$$

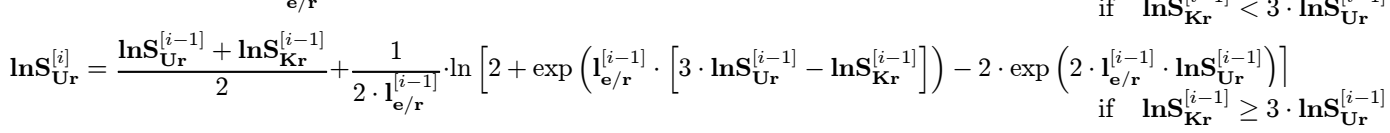

III.3: Calculate bundle survival vectors under linear stress states

$\operatorname{lnS}_{\mathbf{L r}}^{[i]}=\frac{1}{\boldsymbol{\sigma}} \cdot \int^{\boldsymbol{\sigma}} \operatorname{lnS}_{\mathbf{U r}}^{[i]} \cdot \Delta \sigma \quad \operatorname{lnS}_{\mathbf{K} \mathbf{r}}^{[i]}(j)=\frac{k \cdot \ln \mathbf{S}_{\mathbf{L r}}^{[i]}(k \cdot j)-\operatorname{lnS}_{\mathbf{L r}}^{[i]}(j)}{k-1} \quad$ if $\quad j \leq n_{\mathbf{K}} \quad \operatorname{lnS}_{\mathbf{K r}}^{[i]}(j)=\mathcal{C}_{\mathbf{K}} \cdot \ln \mathbf{S}_{\mathbf{U r}}^{[i]}(j) \quad$ if $\quad j>n_{\mathrm{K}}$

\section{Post-processing}

IV.0: Set bundle level counter

$i=0$

$\longrightarrow i=i+1$

IV.1: Calculate bundle strength distributions

$\mathbf{F}_{\mathbf{U r}}^{[i]}=1-\exp \left[\ln \mathbf{S}_{\mathbf{U r}}^{[i]}\right]$

IV.2: Calculate bundle strength statistics

$X_{\mathrm{m}}^{[i]}=\boldsymbol{\sigma}\left(n_{\boldsymbol{\sigma}}\right)-\int \mathbf{F}_{\mathrm{Ur}}^{[i]} \cdot \Delta \sigma$ $\mathrm{CoV}_{X}^{[i]}=\frac{\sqrt{\boldsymbol{\sigma}\left(n_{\boldsymbol{\sigma}}\right)^{2}-\left(X_{\mathrm{m}}^{[i]}\right)^{2}-2 \cdot \int \boldsymbol{\sigma} \cdot \mathbf{F}_{\mathrm{Ur}}^{[i]} \cdot \Delta \sigma}}{X_{\mathrm{m}}^{[i]}}$

Figure 4: Numerical implementation. 


\section{Results}

\subsection{Analysis of model predictions}

Figure 5 summarises the results of the model for nominal input parameters (Table 1). Predicted bundle strength distributions (Figure 5a) evidence size effects, with larger bundles (e.g. $i=15$ ) being weaker but less variable than their constituent fibres $(i=0)$.

In Figure 5b, the model predicts an initial strengthening throughout bundle hierarchy and steep reduction in variability (validated experimentally in Figure 12); these aspects differ from Newman and Gabrielov's (1991) model.

The Weibull plot in Figure 5c shows a concave-down curvature for all $i>0$; this curvature initially increases for small bundles, but is progressively reduced for larger bundles within a $0.01-99.99 \%$ probability range. Figure 5d highlights the asymptotic behaviour of bundle strength distributions (Equations 17 for LTAs and 16 for RTAs); as bundle level increases, these asymptotes are valid for progressively more reduced tails. Such behaviour is common to other FBMs (Harlow and Phoenix, 1978b).

\subsection{Convergence study}

The numerical implementation of the model (Section 2.6) is validated in Figure 6, by studying the convergence of the $\mathrm{CoV}$ of strength distributions (sensitive to both mean value and tails) when $\Delta \sigma \rightarrow 0$ and $\sigma^{\max } \rightarrow \infty$. The effect of using RTAs (Equation 18) in the formulation is noticeable in Figure 6b: the asymptotic relation is activated for $\sigma^{\infty}>\sigma^{\max } / k$, thus resulting in very large errors when $\sigma^{\max }$ is too small for the asymptote to be applicable. Nevertheless, a fully converged set of strength distributions (from the single fibre to a standard coupon-size FRP) is computed in less than a second.

\subsection{Parametric study on fibre and matrix properties}

The relation between single-fibre and bundle strength statistics is shown in Figures 7 and 8. Mean bundle strengths increase as fibres become stronger (Figure 7), with a directly proportional relation for constant fibre-to-matrix strength ratios. Figure 8

Table 1: Nominal model inputs for parametric studies (nominal outputs will be highlighted as $\diamond$ ).

\begin{tabular}{cc|ccccc|ccc|c}
\hline \hline \multicolumn{2}{c|}{ Numerical } & \multicolumn{5}{c|}{ Mechanical properties } & \multicolumn{3}{c|}{ Geometry } & Load \\
\hline$\sigma^{\max }$ & $\Delta \sigma$ & $X_{\mathrm{m}}^{\mathrm{f}}$ & $\mathrm{CoV}_{X}^{\mathrm{f}}$ & $\sigma_{0}^{\mathrm{f}}$ & $m$ & $\tau_{\mathrm{SL}}$ & $\phi^{\mathrm{f}}$ & $V^{\mathrm{f}}$ & $l_{\mathrm{r}}$ & $k$ \\
$(\mathrm{GPa})$ & $(\mathrm{MPa})$ & $(\mathrm{GPa})$ & $(\%)$ & $(\mathrm{GPa})$ & $(-)$ & $(\mathrm{MPa})$ & $(\mu \mathrm{m})$ & $(\%)$ & $(\mathrm{mm})$ & $(-)$ \\
50 & 1 & 4.5 & 25 & 4.93 & 4.54 & 70 & 5 & 60 & 10 & 2 \\
\hline \hline
\end{tabular}




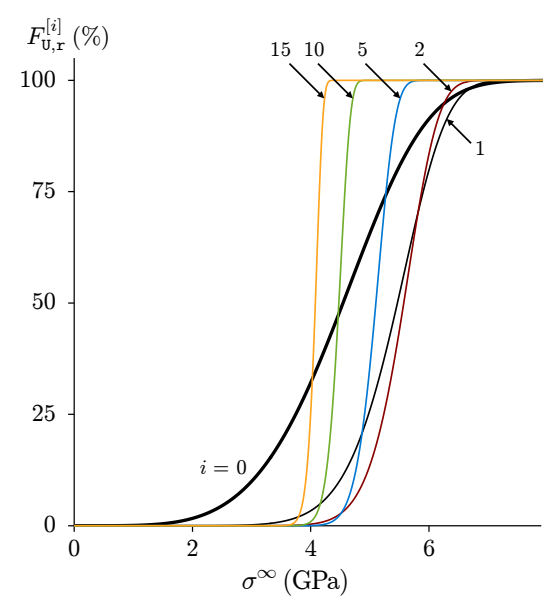

(a) Predicted bundle strength distributions (for several levels $i$ ).

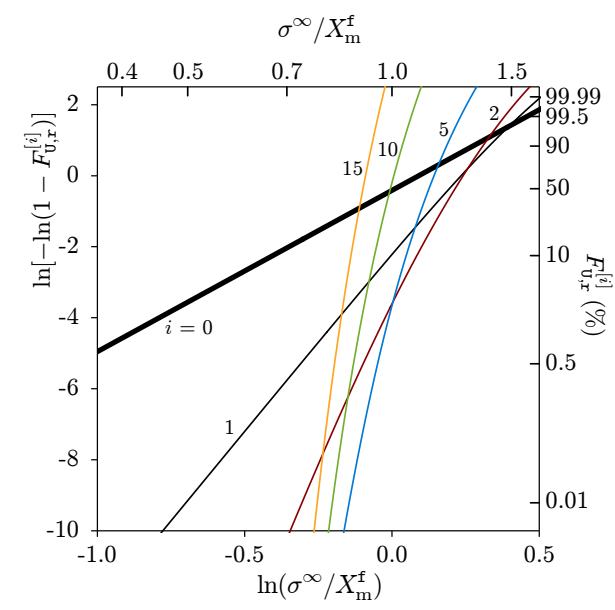

(c) Weibull plot for predicted bundle strength distributions (several levels $i$ ).

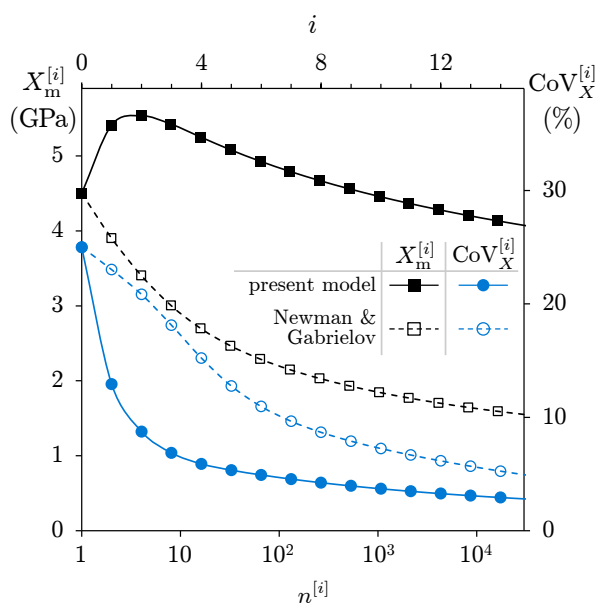

(b) Bundle strength size effect: present model vs. Newman and Gabrielov (1991).

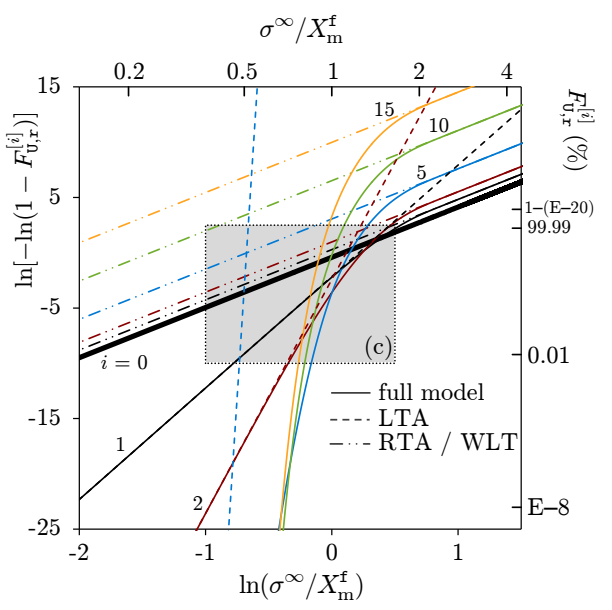

(d) Asymptotic behaviour in Weibull plots (region from plot (c) highlighted).

Figure 5: Overview of model results.

shows that, for a deterministic fibre strength, the model predicts no size effects; for a small $\mathrm{CoV}_{X}^{\mathrm{f}}$ the model converges to the WLT (Equation 16), albeit at a slower rate for large bundles.

Figure 9 shows that increasing the strength of the matrix / interface strengthens the bundles and reduces strength variability. For very low $\tau_{\mathrm{SL}}$ values, bundle strength decreases monotonically with bundle level; as the shear-lag strength becomes negligible $\left(\tau_{\mathrm{SL}} \rightarrow 0\right)$, the model converges to the WLT (Equation 2). 


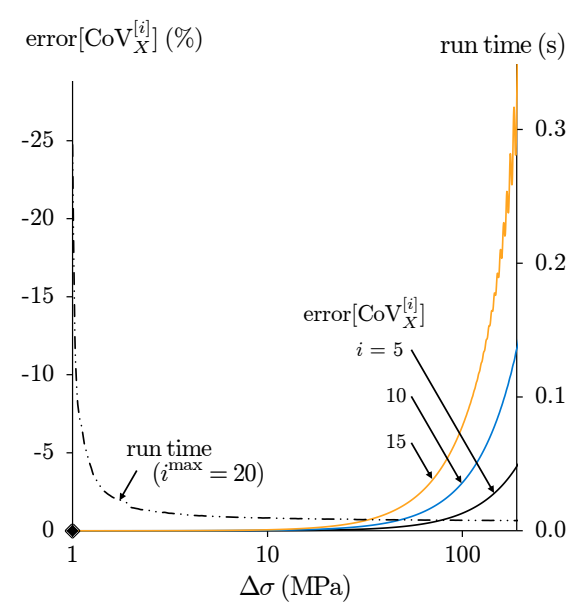

(a) Integration step.

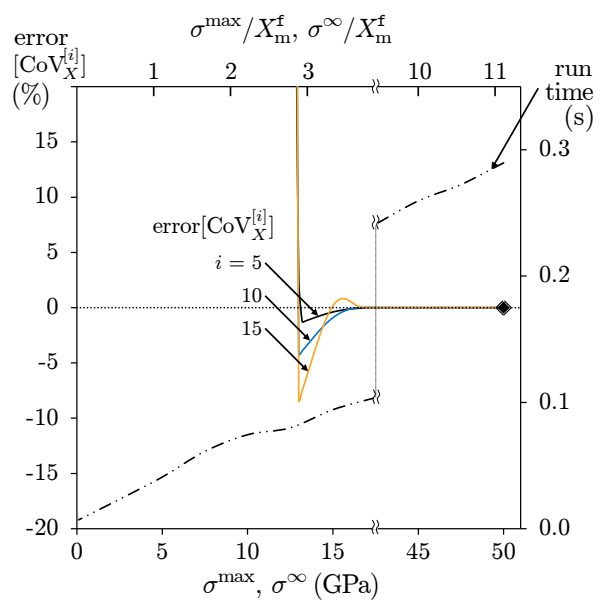

(b) Upper integration limit.

Figure 6: Numerical convergence of the $\mathrm{CoV}$ of strength distributions (for several levels $i$ ).

Errors are relative to nominal inputs (Table 1), and run times were obtained with an Intel(R) Core(TM)2 Quad CPU @ $2.50 \mathrm{GHz}$, for $i^{\max }=20\left(n^{[i]} \approx 10^{6}\right)$.

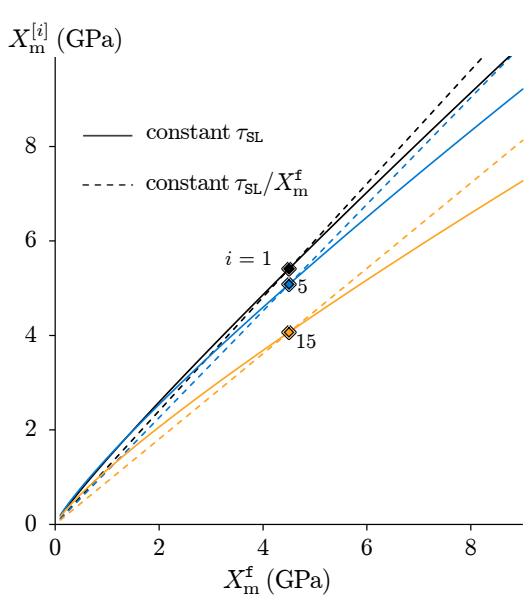

(a) Mean bundle strength.

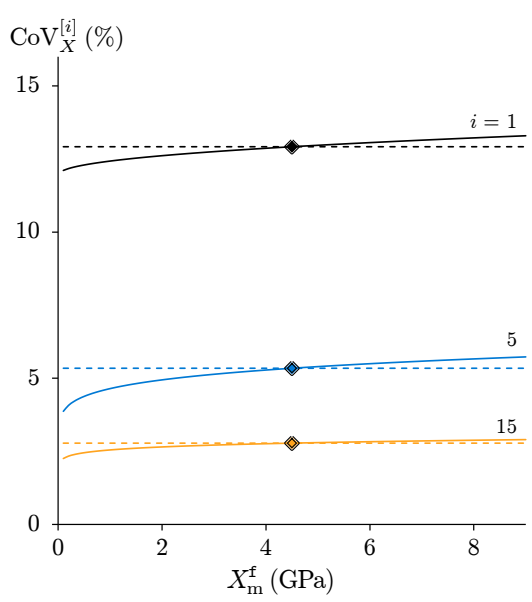

(b) Bundle strength $\mathrm{CoV}$.

Figure 7: Effect of mean single-fibre strength on bundle strength statistics (for several levels $i$ ).

\subsection{Asymptotic limits}

Varying the stress concentrations factor $k$ (Figure 10) shows the model is bounded by the WLT for $k \rightarrow \infty$. For $k=1$, a Strongest Link Theory (SLT) applies (a level- $[i+1]$ bundle fails within $l_{\mathrm{c}}^{[i+1]}$ only if no level-[i] bundle survives).

Figure 11 compares bundle strengths obtained by either (i) running the full model for all bundle levels $i$, or (ii) running the model up to level $i^{\mathrm{WLT}}$ followed by the WLT for levels $i>i^{\mathrm{WLT}}$ (Equation 1, with $n=2^{i-i^{\mathrm{WLT}}}$ ). Although the WLT applied directly to single-fibre $\left(i^{\mathrm{WLT}}=0\right)$ is extremely inaccurate, both approaches converge if applied 


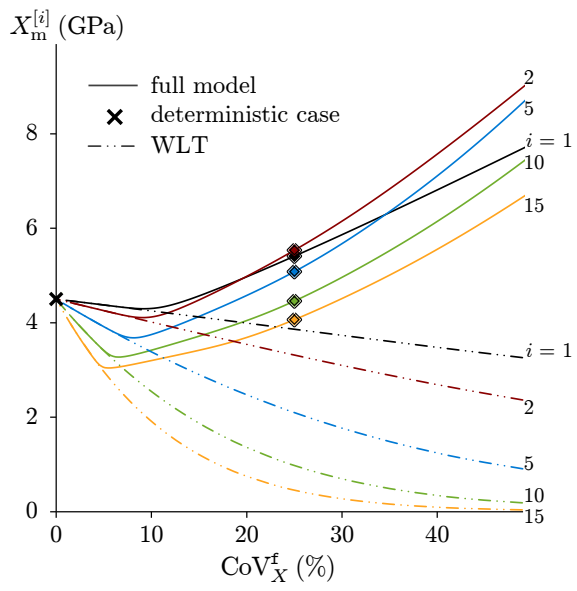

(a) Mean bundle strength.

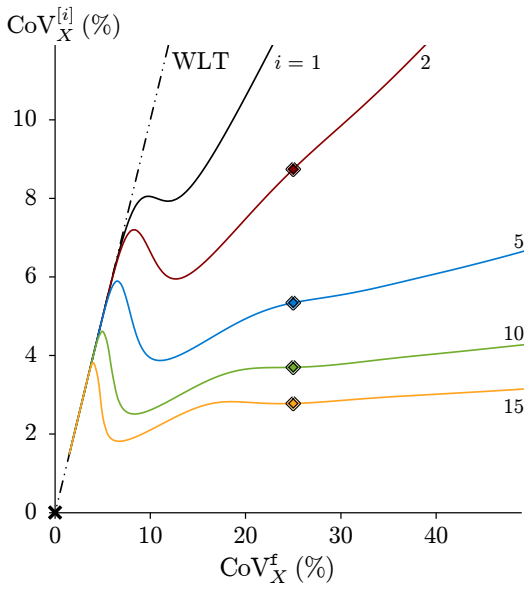

(b) Bundle strength CoV.

Figure 8: Effect of the $\mathrm{CoV}$ of single-fibre strength on bundle strength statistics (for several levels $i$ ).

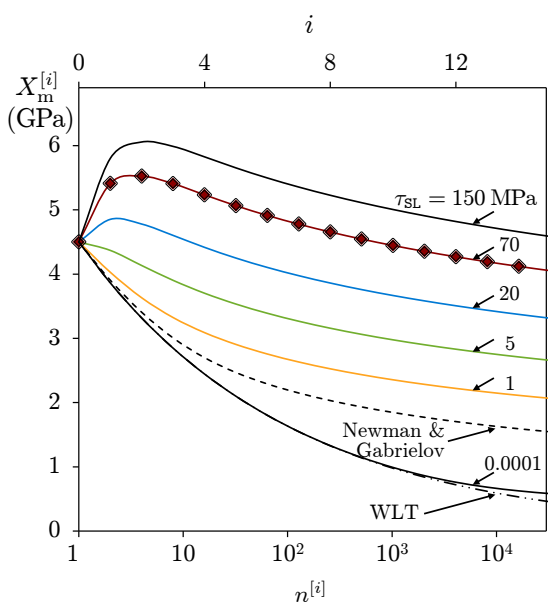

(a) Mean bundle strength.

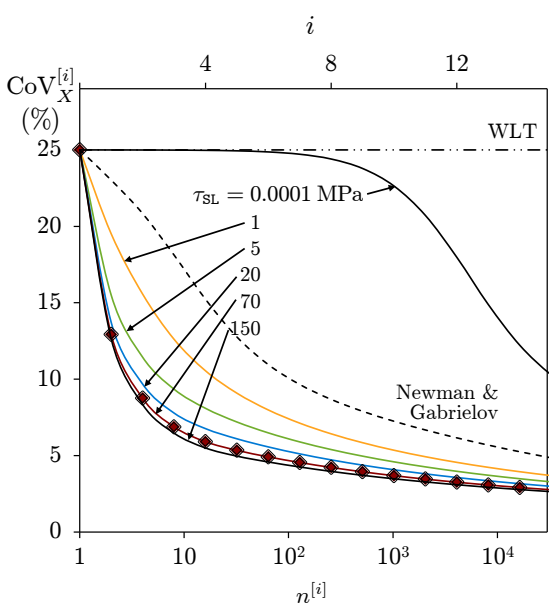

(b) Bundle strength CoV.

Figure 9: Bundle strength size effect for several shear-lag strengths $\left(\tau_{\mathrm{SL}}\right)$, and comparison with Newman and Gabrielov's (1991) model and WLT.

from a certain bundle level onwards (in this case, for $i^{\mathrm{WLT}} \gtrsim 5$ ).

\subsection{Validation against experimental results}

\subsubsection{Micro-composites}

Figure 12 validates the model against the experiments from Beyerlein and Phoenix (1996a) and Kazanci (2004) on micro-composites, combining different carbon fibres, epoxy matrices and bundle geometries. Materials and input properties are shown in Tables 2 and 3; single-fibre distributions were fitted as in Figure 12. Free-edge effects (configuration QB, Appendix A) and a stress concentration factor $k=2$ were considered; 


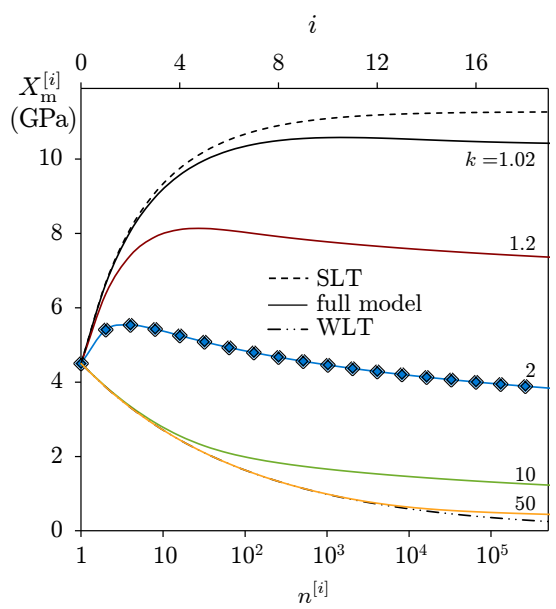

(a) Mean bundle strength.

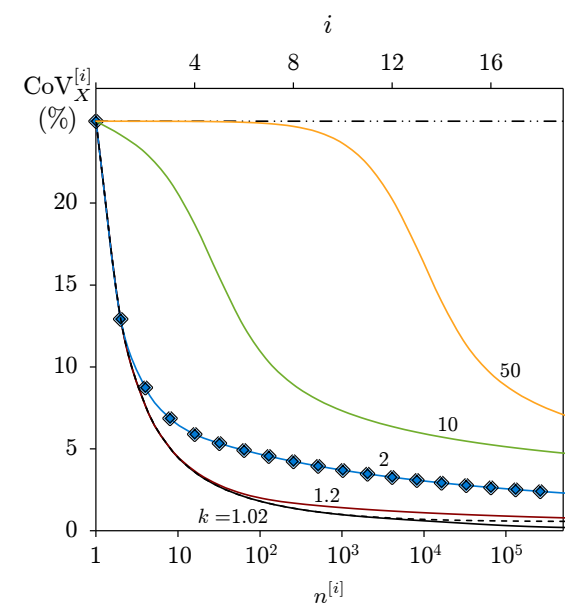

(b) Bundle strength CoV.

Figure 10: Bundle strength size effect for different values of the stress concentrations factor $k$, highlighting the asymptotic limits to the WLT $(k \rightarrow \infty)$ and to the SLT $(k=1)$.

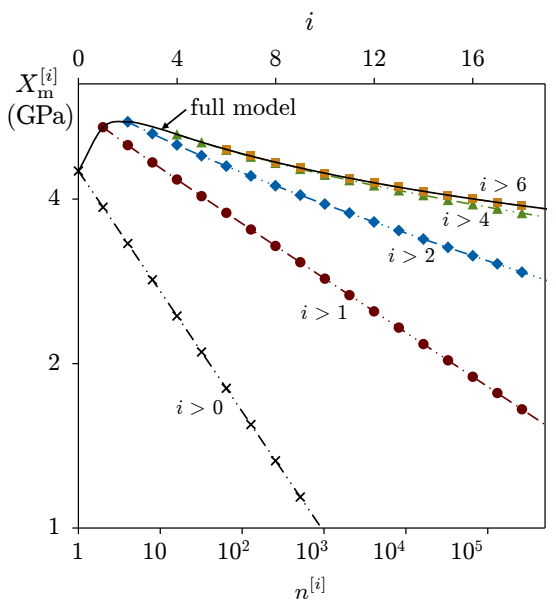

(a) Mean bundle strength.

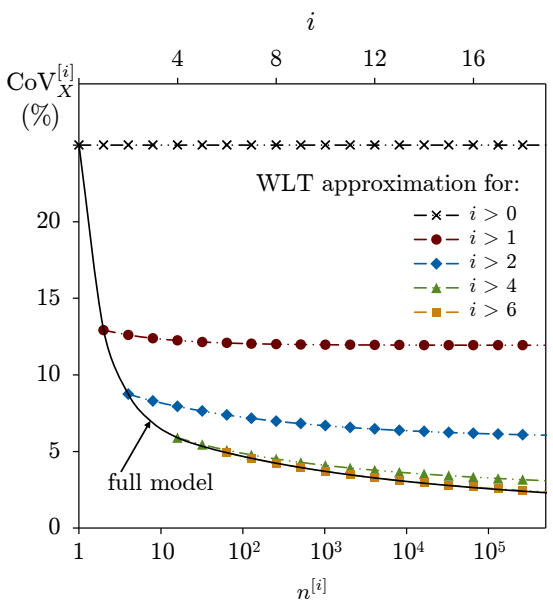

(b) Bundle strength CoV.

Figure 11: Bundle strength size effect considering a WLT approximation for large bundles. Note that, for $i^{\mathrm{WLT}}>0$, size effects on the mean strength are not linear in the log-log plot.

strength distributions were obtained directly at $i=2$ for the 4 -fibres bundles, and by spline interpolation for the 7-fibres bundles. Each plot presents strength distributions for a fibre type and corresponding bundles (with two different resins); predictions from Newman and Gabrielov's (1991) model are shown for comparison.

\subsubsection{Macro-composites}

Okabe and Takeda (2002) analysed size effects on the strength of a Toray T800H/3631 (carbon-epoxy) system (Tables 2 and 3) by testing single fibres of several lengths, as 
Table 2: Description of composites for model validation.

\begin{tabular}{ccccccl}
\hline \hline Ref. & $\begin{array}{c}\text { Fibre } \\
\text { ref. }{ }^{(\star)}\end{array}$ & $n^{[i]}$ & $\begin{array}{c}\text { Matrix } \\
\text { ref. }{ }^{(\dagger)}\end{array}$ & $\begin{array}{c}\tau_{\mathrm{SL}} \\
(\mathrm{MPa})\end{array}$ & $V^{\mathrm{f}}$ & \multicolumn{1}{c}{ Reference } \\
\hline $\mathrm{A}^{4} \mathrm{~S}$ & $\mathrm{~A}$ & 4 & $\mathrm{~S}$ & $46.6^{(\S)}$ & $70 \%$ & Beyerlein and Phoenix (1996a) \\
$\mathrm{A}^{4} \mathrm{~F}$ & $\mathrm{~A}$ & 4 & $\mathrm{~F}$ & $10.3^{(\S)}$ & $70 \%$ & Beyerlein and Phoenix (1996a) \\
$\mathrm{I}^{7} \mathrm{~S}$ & $\mathrm{I}$ & 7 & $\mathrm{~S}$ & $46.6^{(\S)}$ & $56 \%$ & Kazanci (2004) \\
$\mathrm{I}^{7} \mathrm{~F}$ & $\mathrm{I}$ & 7 & $\mathrm{~F}$ & $10.3^{(\S)}$ & $56 \%$ & Kazanci (2004) \\
\hline $\mathrm{T}^{\mathrm{n}} \mathrm{T}$ & $\mathrm{T}$ & $10^{4}-10^{6}$ & $\mathrm{~T}$ & 52.4 & $60 \%$ & Okabe and Takeda (2002) \\
\hline \hline
\end{tabular}

(*) See Table 3 for detailed description.

(†) Epoxy resins. Standard (S): DER 331, Dow Plastics; Flexible (F): DER 331 + DER 732 (50:50), Dow Plastics; Toughened (T): 3631, Toray Composites.

(§) Drucker-Prager's criterion, using tensile and compressive strengths (Dow Plastics, 1999).

Table 3: Carbon-fibre data for model validation.

\begin{tabular}{ccccccl}
\hline \hline $\begin{array}{c}\text { Fibre } \\
\text { ref. }\end{array}$ & $\begin{array}{c}\text { Fibre } \\
\text { type }\end{array}{ }^{(\star)}$ & $\begin{array}{c}\phi^{\mathrm{f}} \\
(\mu \mathrm{m})\end{array}$ & $\begin{array}{c}l_{\mathrm{r}}^{\mathrm{f}} \\
(\mathrm{mm})\end{array}$ & $m^{(\dagger)}$ & $\begin{array}{c}\sigma_{0}^{\mathrm{f}}(\dagger) \\
(\mathrm{GPa})\end{array}$ & \multicolumn{1}{c}{ Reference } \\
\hline $\mathrm{A}$ & $\mathrm{AS} 4$ & 6.85 & 10 & 4.8 & 4.493 & Beyerlein and Phoenix (1996a) \\
$\mathrm{I}$ & $\mathrm{IM} 6$ & 5.63 & 10 & 5.4 & 5.283 & Kazanci (2004) \\
\hline $\mathrm{T}$ & $\mathrm{T} 800$ & 5.00 & 50 & 3.8 & 3.570 & Okabe and Takeda (2002) \\
\hline \hline
\end{tabular}

${ }^{\star}$ ) AS4 and IM6 fibres provided by Hercules / Hexcel; T800 fibres provided by Toray.

(†) From the respective Reference, originally calculated through the maximum likelihood method.

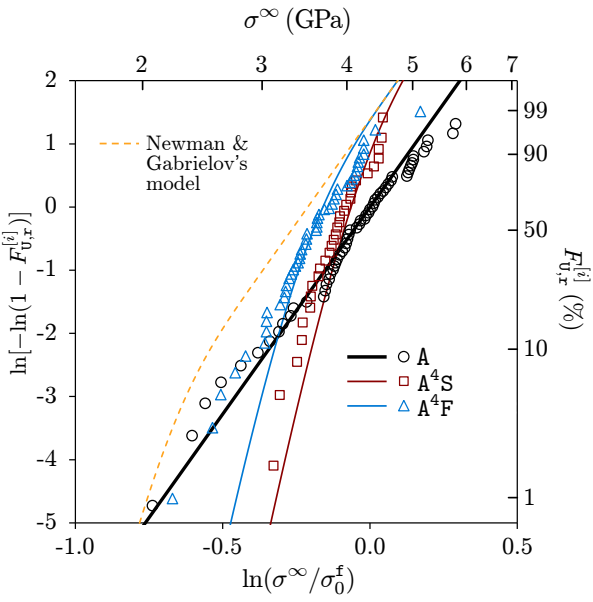

(a) AS4 fibre type

$\left(m=6.6, \sigma_{0}^{\mathrm{f}}=4.3 \mathrm{GPa}\right.$ at $\left.l_{\mathrm{r}}=10 \mathrm{~mm}\right)$.

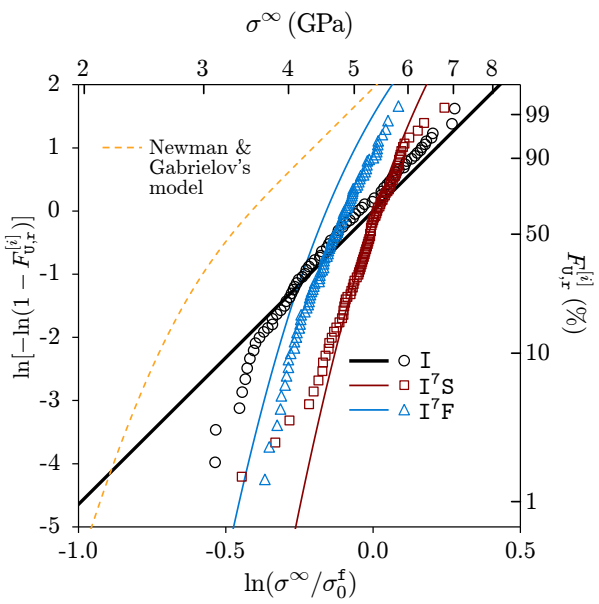

(b) IM6 fibre type

$\left(m=4.6, \sigma_{0}^{\mathrm{f}}=5.3 \mathrm{GPa}\right.$ at $\left.l_{\mathrm{r}}=10 \mathrm{~mm}\right)$.

Figure 12: Micro-composite strength distributions: experimental results (data points from Beyerlein and Phoenix, 1996a, Kazanci, 2004), visually fitted single-fibre distribution for model input (thick lines), present model predictions for both resins types (thin lines), and Newman and Gabrielov's (1991) model prediction (dashed lines). 

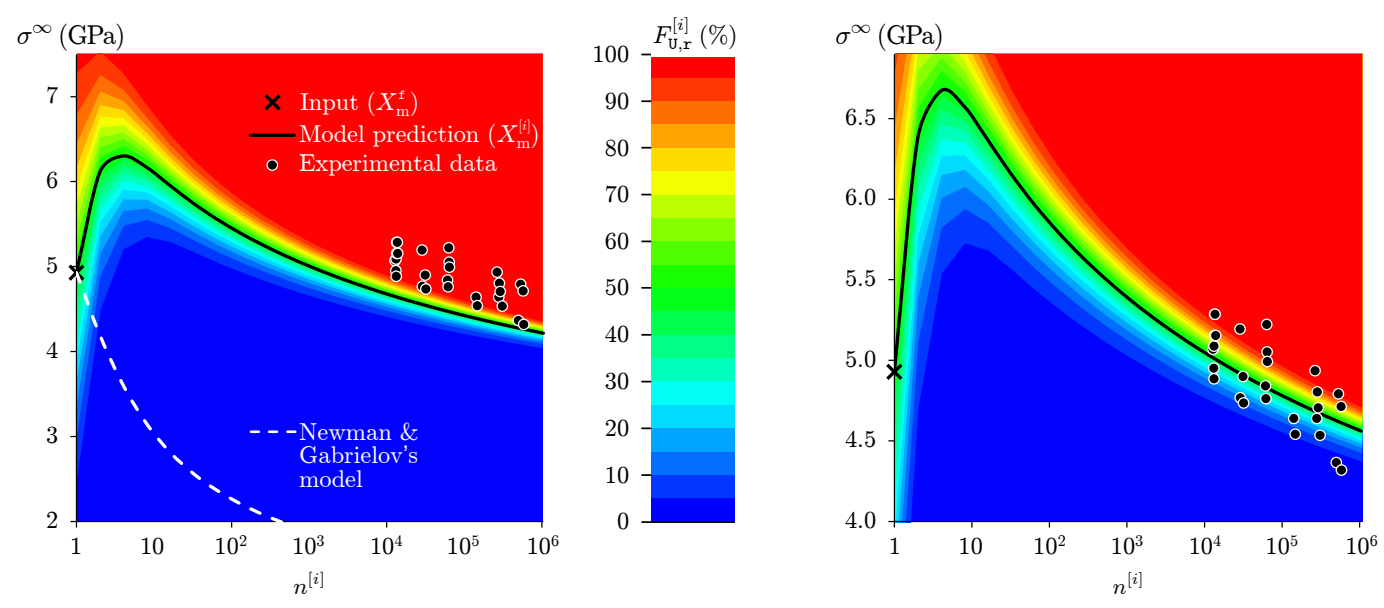

(a) $\tau_{\mathrm{SL}}=52.4 \mathrm{MPa}$ (nominal).

(b) $\tau_{\mathrm{SL}}=79.6 \mathrm{MPa}(50 \%$ stronger $)$.

Figure 13: Macro-bundle strengths for model validation: experimental bundle strengths (data points from Okabe and Takeda, 2002), present model's probability map (with mean strength highlighted), and Newman and Gabrielov's (1991) mean strength prediction (dashed line in (a)).

well as $10 \mathrm{~mm}$ long bundles with $10^{4}-10^{6}$ fibres. Figure 13 shows the experimentally measured bundle strengths, together with the strength probability map predicted by the model (the input data used can be found in Tables 2 and 3, and $k=2$ ); two shearlag strengths are considered: one at the nominal value (Figure 13a), and the other $50 \%$ higher (Figure 13b). Mean bundle strengths predicted by Newman and Gabrielov's (1991) model are shown for comparison.

\section{Discussion}

\subsection{Physically meaningful model features and experimental validation}

The model captures many characteristic features of size-effects in the strength of FRPs:

a. Size effects result from fibre strength variability: Figure 8 shows no size effect for deterministic fibre strength. The WLT governs damage initiation, but propagation is constrained by the matrix / fibre-matrix interface, which limits stress concentrations and coalescence of fibre breaks.

b. Both the magnitude of size effects and the variability of tensile strength decrease with increasing specimen size (Wisnom, 1999), as shown by the upwards curvature of $X_{\mathrm{m}}^{[i]}\left(n^{[i]}\right)$ and $\operatorname{CoV}_{X}^{[i]}\left(n^{[i]}\right)$ in Figure 5b (for $i \gtrsim 3$ ). 
c. The matrix / interface is most important for the micro-scale $(i \lesssim 3)$. For most reasonable $\tau_{\mathrm{SL}}$, small bundles are stronger than single-fibres (Figures 9a and 12b). The local geometry influences the strength of small bundles only (Appendix A).

d. Size effects at the macro-scale $\left(n^{[i]} \gtrsim 50\right)$ are governed by the WLT (Figure 11), which is consistent with the quasi-brittle nature of FRPs (Okabe and Takeda, 2002, Scott et al., 2011, Wisnom, 1999). A critical fibre break cluster - after which failure is catastrophic - is defined by the bundle size at which full model and WLT start converging $\left(n^{[i]} \approx 50\right.$ for the nominal inputs in Table 1$)$.

e. Within a 0.01 - 99.99\% probability range, predicted large-bundle strength distribution appear quasi-linear in a Weibull plot (Figure 5c, $i=15$ ). This agrees with the good fitting usually verified between the Weibull-based WLT and experimental strength distributions and size effects in FRP coupons (Wisnom, 1999).

f. The model replicates accurately the effect of different fibres and resins on microbundle strength distributions (Figure 12); this offers a strong support to the shearlag approach used. The model reproduces the concave-down curvature of bundle strength distributions, the different slopes and locations of the four data sets, and the relative orientations within each pair of data for the same fibre type (converging right tails in Figure 12a, nearly parallel distributions in 12b).

g. Figure $13 \mathrm{~b}$ evidences a good agreement between predicted and measured strengths in the macro scale (cross sections up to $30 \mathrm{~mm}^{2}$, larger than a standard UD FRP tensile-strength specimen, are shown); this implied using a higher shear-lag strength, which is likely to be more representative of the true in-situ matrix behaviour (Gulino et al., 1991, Hobbiebrunken et al., 2007).

\subsection{Relation between the present model and others in the literature}

\subsubsection{Weakest link theory}

The present formulation differs from the WLT by considering stable modes of bundle failure (sequences of events $E_{2}$ and $E_{3}$ in Section 2.3). Consequently, the scaling law (Equation 11) degenerates into the WLT (i.e. $\left.S_{\mathrm{U}, \mathrm{c}}^{[i+1]}\left(\sigma^{\infty}\right) \rightarrow S_{\mathrm{U}, \mathrm{e}}^{[i]}\left(\sigma^{\infty}\right)^{4}\right)$ whenever these failure modes cannot take place:

a. Low $\operatorname{CoV}_{X}^{\mathrm{f}}$ (Figure 8, $\mathcal{C}_{\mathrm{K}} \geq 3$ ) and / or $k \rightarrow \infty$ (Figure 10). Here, the weakest fibre's neighbour cannot withstand the stress concentrations after first failure; 
b. $\tau_{\mathrm{SL}} \rightarrow 0$ (Figure 9 ). As the scaling law is formulated at the length $l_{\mathrm{c}}^{[i+1]} \propto 1 / \tau_{\mathrm{SL}}$, this case degenerates into a dry (loose) bundle with infinite length under local load sharing, governed by the WLT;

c. Large $i$ or $n^{[i]}$ (Figure 11). As strength variability decreases with increasing bundle size (Figure 5b), failure of a sufficiently large sub-bundle immediately triggers failure of its neighbour.

\subsubsection{Newman and Gabrielov's (1991) model}

The present model shows key differences to the one originally developed by Newman and Gabrielov (1991, Equation 3):

a. This model considers a shear-lag boundary; this confines stress concentrations around fibre breaks, and defines a characteristic length $\left(l_{\mathrm{c}}^{[i]}\right.$, generally shorter than the original bundle length). Consequently, the present model predicts higher mean bundle strength and lower variability (Figure 5b);

b. As the characteristic length is fundamental for size effects in quasi-brittle materials (Bažant, 1999), the present work shows a much better correlation with experiments in FRPs (Figures 12 and 13) than the original model from Newman and Gabrielov, which was developed for dry fibre bundles;

c. Such characteristic length makes this model consistent with the WLT for length scaling. On the contrary, applying Newman and Gabrielov's (1991) model to bundles of different lengths yields strength distributions which cannot be related by the WLT.

d. For extremely weak matrices, the present model converges not to Newman and Gabrielov's model directly, but to its asymptotic limit for large bundles — the WLT (Figure 9). This is again due to using $l_{\mathrm{c}}^{[i+1]} \propto 1 / \tau_{\mathrm{SL}}$ as characteristic length.

e. Newman and Gabrielov reduced the order of their scaling law by re-organising the computations. Instead, this was addressed in the present model by using an asymptotic result (Equation 18), which would be applicable to the former model as well.

\subsubsection{Fibre-bundle models}

The central aspects relating the present model and other fibre-bundle models in the literature are discussed below: 
a. Analytical fibre-bundle models with local load sharing become prohibitively complex for bundles with more than 10 fibres (Harlow and Phoenix, 1978a); this has been overcome in the literature through asymptotic simplifications (Curtin, 1993, Phoenix et al., 1997, 1988, Phoenix and Smith, 1983) and Monte-Carlo simulations (Landis et al., 2000, Mahesh et al., 2002, Okabe and Takeda, 2001, Wada and Fukuda, 1999). The present model is made suitable to a complete range of scales (Figure 5) by the use of hierarchies and an efficient implementation scheme; still, the typical features of fibre bundle models are captured - namely size effects and the existence of a critical cluster of fibre breaks (Figure 11).

b. Several authors (Behzadi et al., 2009, Landis et al., 2000, Mahesh et al., 2002, Phoenix et al., 1988) recognised that, although their models used a fixed characteristic length, this is not realistic nor accurate; Wisnom and Green (1995) considered the characteristic length to be additive regarding the number of broken fibres, but included no stress concentrations in their calculations. The present model uses a variable control length $l_{\mathrm{c}}^{[i+1]}$ (Equation 12) that depends on the level of the broken cluster; this is in agreement with experimental observations of self-similar fracture surfaces in FRPs (Laffan et al., 2010, Pimenta et al., 2010).

c. This model uses simple definitions of load transfer and characteristic length (Equation 12), as well as a constant stress concentration factor $(k=2$ from equilibrium of forces). Other definitions can be explored, namely on the matrix response (Nairn, 1997, Okabe and Takeda, 2001), the size of $l_{\mathrm{c}}$ (Phoenix et al., 1997), and magnitude of stress concentrations (Beyerlein and Phoenix, 1996b, Hedgepeth, 1961, Nedele and Wisnom, 1994). However, these are still unresolved topics in the literature, hence justifying the use of simplified approaches.

\section{Conclusions}

An analytical model for size effects on the longitudinal tensile strength of FRP bundles was developed, implemented and validated. The model is based on the stochastic analysis of the failure process in hierarchical fibre bundles, considering Weibull fibre-strength distributions. Matrix effects are represented through a simplified shear-lag model, so that the control length (in which fibre breaks interact) scales hierarchically as well. 
The model predicts full strength distributions and statistics for bundles of any size. The matrix (or fibre-matrix interface) was shown to have a significant strengthening effect, which supports the present model over others not including this feature (e.g. WLT and Newman and Gabrielov, 1991). An efficient numerical scheme was proposed, leading to full-model running times below one second.

The model was validated both at the micro and macro scales, showing a remarkable agreement with experimentally measured bundle strengths in a large range of sizes. The quasi-brittle nature of composites is reproduced; the model also illustrates many experimentally observed trends, such as the tensile strength of FRPs appearing to follow a Weibull distribution, and large-scale size effects consistent with the WLT.

Predictive models for size effects in composite materials are paramount for scaling small-coupon experimental results to the design of large structures. In addition to such quantitative predictions, the present work provides insight on the longitudinal tensile failure process. The model's ability to compute strength distributions for small bundles (rather than only for asymptotically large ones) makes it particularly suitable for state-of-the-art multiscale discontinuous-fibre composites. This model has also been applied to predict the fracture toughness of FRPs under longitudinal tensile failure (Pimenta and Pinho, 2013).

\section{Acknowledgements}

The funding from the Portuguese Foundation for Science and Technology (project nr. SFRH/BD/44051/2008) is gratefully acknowledged.

\section{Appendix A. Effect of bundle and shear-lag boundary geometry on strength}

Table A.1 defines alternative geometries for the shear-lag boundary, taking into account different fibre arrangements, failure paths and free-edge effects.

Matching the perimeter of these different geometries with the corresponding mean bundle strength (Figure A.1) reveals that the largest shear-lag boundary at low-level bundles (QM geometry) yields the strongest bundles throughout the whole hierarchy. On the contrary, a large boundary at high-level bundles (HI geometry) yields no strengthening effect. Free-boundary effects (QB vs. QI geometries) affect small bundles only.

All these observations support that (i) the shear-lag boundary plays a critical role

for the strength of small-scale bundles, while (ii) the weakest link theory dominates size effects for large-scale bundles (see Section 4.1 for further discussion). 
Table A.1: Alternative definitions of the shear-lag boundary.

Perimeters in square arrangement were derived for even values of $i$ (with $n^{[i]}=2^{i}$ ); perimeters in hexagonal arrangement were derived for integer values of the hexagonal level $i_{\mathrm{H}}$ (with $n^{[i]}=7^{i_{\mathrm{H}}}=2^{i}$ ).

\begin{tabular}{ll}
\hline \hline Ref. & Description and perime \\
\hline QM & Square fibre arrangement, preferential \\
& $C_{\mathrm{QM}}^{[i]}=4 \cdot \sqrt{n^{[i]}} \cdot l_{\mathrm{Q}} \quad$ with $\quad l_{\mathrm{Q}}=\frac{\sqrt{\pi} \cdot \phi^{\mathrm{f}}}{2 \cdot \sqrt{V^{\mathrm{f}}}}$
\end{tabular}

Diagram

QI Square fibre arrangement, preferential interfacial failure (default configuration, Equation 5 in Section 2.1):

$C_{\mathrm{QI}}^{[i]}=3 \cdot C^{\mathrm{f}}+4 \cdot\left[\left(\sqrt{n^{[i]}}-1\right) \cdot s_{\mathrm{Q}}+\left(\sqrt{n^{[i]}}-2\right) \cdot \frac{C^{\mathrm{f}}}{2}\right]$

QS Square fibre arrangement, shortest failure path:

$C_{\mathrm{QS}}^{[i]}=C^{\mathrm{f}}+4 \cdot\left(\sqrt{n^{[i]}}-1\right) \cdot l_{\mathrm{Q}}$

HI Hexagonal fibre arrangement, preferential interfacial failure (fractal boundary, hence initial recursive relation):

$$
\begin{aligned}
& C_{\mathrm{HI}}^{\left[i_{\mathrm{H}}\right]}=6 \cdot\left(\frac{C_{\mathrm{HI}}^{\left[i_{\mathrm{H}}-1\right]}}{2}+\frac{C^{\mathrm{f}}}{6}+s_{\mathrm{H}}\right) \text { with } s_{\mathrm{H}}=\left(\sqrt{\frac{\pi}{2 \sqrt{3} V^{\mathrm{f}}}}-1\right) \cdot \phi^{\mathrm{f}} \\
& C_{\mathrm{HI}}^{\left[i_{\mathrm{H}}\right]}=3^{i_{\mathrm{H}}} \cdot C^{\mathrm{f}}+\frac{3^{i_{\mathrm{H}}}-1}{2} \cdot\left(C^{\mathrm{f}}+6 \cdot s_{\mathrm{H}}\right) \\
& C_{\mathrm{HI}}^{\left[i_{\mathrm{H}}\right]} \equiv C_{\mathrm{HI}}^{[i]}=3 \cdot\left(\sqrt[\log _{3} \sqrt[7]{n^{[i]}}]{[}-1\right) \cdot s_{\mathrm{H}}+\left(3 \cdot \sqrt[\log _{3}]{n^{[i]}}-1\right) \cdot \frac{C^{\mathrm{f}}}{2}
\end{aligned}
$$

QB Square fibre arrangement, preferential interfacial failure, considering free-edge effects in levels $i^{\max }-2$ and $i^{\max }-1$ (configuration QI should be used up to level $i^{\max }-3$ ):

$$
\begin{aligned}
& C_{\mathrm{QB}, \text { edge }}^{\left[i_{\max }-1\right]}=\sqrt{2 \cdot n^{[i]}} \cdot\left(s_{\mathrm{Q}}+\frac{C^{\mathrm{f}}}{2}\right) \text { with } s_{\mathrm{Q}}=\left(\frac{\sqrt{\pi}}{2 \sqrt{V^{\mathrm{f}}}}-1\right) \cdot \phi^{\mathrm{f}} \\
& C_{\mathrm{QB}, \text { corner }}^{\left[i_{\max }-2\right]}=\frac{C^{\mathrm{f}}}{4}+2 \cdot\left(\sqrt{n^{[i]}}-\frac{1}{2}\right) \cdot\left(s_{\mathrm{Q}}+\frac{C^{\mathrm{f}}}{2}\right)
\end{aligned}
$$
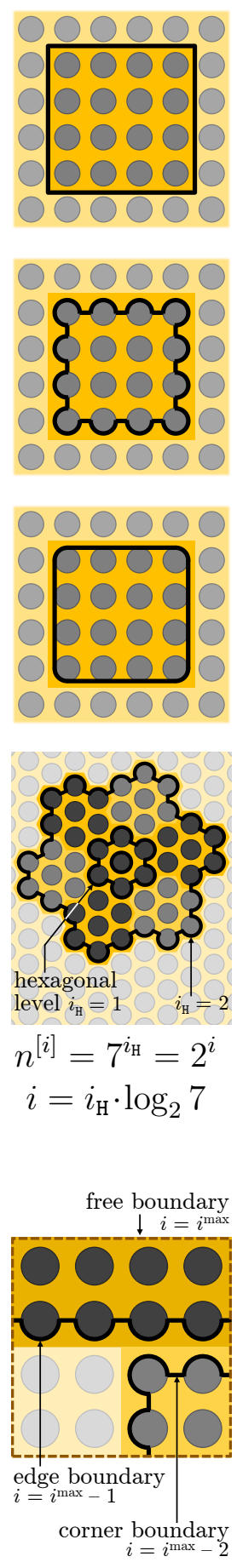


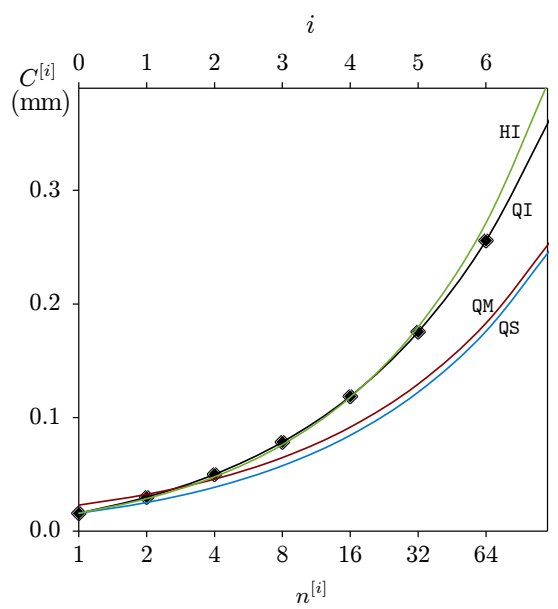

(a) Shear-lag boundary perimeter.

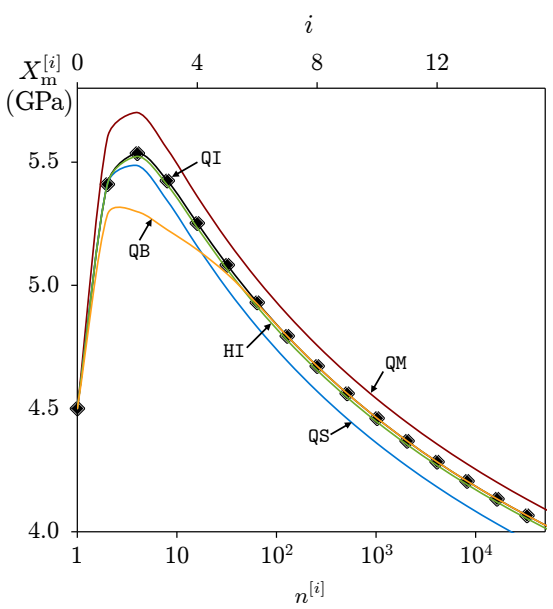

(b) Mean bundle strength.

Figure A.1: Bundle strength size effect for several shear-lag geometries.

\section{Appendix B. Generalisation of the weakest link theory to non-uniform stress fields}

The weakest link theory (Equation 1) can be generalised to non-uniform stress fields. Consider a chain composed by $n$ elements, each of length $l_{\mathrm{r}}$ and under a uniform tensile stress $\sigma_{j}, j=\{1 \ldots n\}$. The chain (of length $l_{\mathrm{n}}=n \cdot l_{\mathrm{r}}$ ) is subjected to a piecewise constant but otherwise generic stress field $\Phi$, with survival probability $S_{\Phi, \mathrm{n}}$ related to those of the uniformly loaded element $\left(S_{\mathrm{U}, \mathrm{r}}\right)$ and the chain with same length $\left(S_{\mathrm{U}, \mathrm{n}}\right)$ :

$$
S_{\Phi, \mathrm{n}}=\prod_{j=1}^{n} S_{\mathrm{U}, \mathrm{r}}\left(\sigma_{j}\right) \stackrel{\text { Eq. } 1}{\longrightarrow} \ln \left[S_{\Phi, \mathrm{n}}\right]=\sum_{j=1}^{n} \frac{l_{\mathrm{r}}}{l_{\mathrm{n}}} \cdot \ln \left[S_{\mathrm{U}, \mathrm{n}}\left(\sigma_{j}\right)\right]
$$

This relation can be applied to a fibre or bundle of length $l$ under a linear stress field (defined by the variable remote stress $\sigma^{\infty}$ and fixed stress concentration factor $k$, e.g. segment $\mathcal{B}_{1}$ in Figure 3a):

$$
\sigma_{\mathrm{K}}(x)=\sigma^{\infty}+\frac{\sigma^{\infty} \cdot(k-1)}{l} \cdot x \quad, \quad x \in[0, l]
$$

Dividing the chain into $n \rightarrow \infty$ links of length $\Delta x=l / n$, the survival probability $S_{\mathrm{K}}$ of the chain under $\sigma_{\mathrm{K}}(x)$ relates to that of a uniformly loaded chain $\left(S_{\mathrm{U}}\right)$ by:

$$
\ln \left[S_{\mathrm{K}}\right]=\lim _{n \rightarrow \infty} \sum_{j=1}^{n} \frac{\Delta x}{l} \cdot \ln \left[S_{\mathrm{U}}\left(\sigma_{\mathrm{K}}\left(x_{j}\right)\right)\right]=\frac{1}{l} \int_{x=0}^{l} \ln \left[S_{\mathrm{U}}\left(\sigma_{\mathrm{K}}(x)\right)\right] \mathrm{d} x
$$


Changing the integration variable from $x$ to $\sigma_{\mathrm{K}}$ (using the field in Equation B.2),

$$
\ln \left[S_{\mathrm{K}}\left(\sigma^{\infty}\right)\right]= \begin{cases}\frac{1}{\sigma^{\infty} \cdot(k-1)} \int_{\sigma_{\mathrm{K}}=\sigma^{\infty}}^{k \cdot \sigma^{\infty}} \ln \left[S_{\mathrm{U}}\left(\sigma_{\mathrm{K}}\right)\right] \mathrm{d} \sigma_{\mathrm{K}} & \text { if } \quad k>1 \\ \ln \left[S_{\mathrm{U}}\left(\sigma^{\infty}\right)\right] & \text { if } \quad k=1\end{cases}
$$

A similar procedure can be applied to a pure linear stress field $\sigma_{\mathrm{L}}(x)$, under which the chain survival probability is then $S_{\mathrm{L}}$ :

$$
\sigma_{\mathrm{L}}(x)=\frac{\sigma^{\infty}}{l} \cdot x, \quad x \in[0, l] \quad \Rightarrow \quad \ln \left[S_{\mathrm{L}}\left(\sigma^{\infty}\right)\right]=\frac{1}{\sigma^{\infty}} \int_{\sigma_{\mathrm{L}}=0}^{\sigma^{\infty}} \ln \left[S_{\mathrm{U}}\left(\sigma_{\mathrm{L}}\right)\right] \mathrm{d} \sigma_{\mathrm{L}}
$$

Combining both previous equations, $S_{\mathrm{K}}\left(\sigma^{\infty}\right)$ can also be defined by:

$$
\ln \left[S_{\mathrm{K}}\left(\sigma^{\infty}\right)\right]= \begin{cases}\frac{k \cdot \ln \left[S_{\mathrm{L}}\left(k \cdot \sigma^{\infty}\right)\right]-\ln \left[S_{\mathrm{L}}\left(\sigma^{\infty}\right)\right]}{k-1} & \text { if } \quad k>1 \\ \ln \left[S_{\mathrm{U}}\left(\sigma^{\infty}\right)\right] & \text { if } \quad k=1\end{cases}
$$

Equations B.4-B.6 are valid regardless of the shape of the strength distribution $S_{\mathrm{U}}\left(\sigma^{\infty}\right)$. For the particular case of a Weibull distribution with parameters $m$ and $\sigma_{0}$,

$$
\begin{array}{r}
\ln \left[S_{\mathrm{K}}\left(\sigma^{\infty}\right)\right]=\mathcal{C}_{\mathrm{K}} \cdot \ln \left[S_{\mathrm{U}}\left(\sigma^{\infty}\right)\right] \Rightarrow S_{\mathrm{K}}\left(\sigma^{\infty}\right)=\exp \left[-\mathcal{C}_{\mathrm{K}} \cdot\left(\frac{\sigma^{\infty}}{\sigma_{0}}\right)^{m}\right] \\
\text { where } \quad \mathcal{C}_{\mathrm{K}}=\frac{k^{m+1}-1}{(m+1)(k-1)}
\end{array}
$$

\section{Appendix C. Physically-based derivation of the hierarchical scaling law}

The scaling law in Equation 8 can be derived from the physically reasonable sequences of events $E_{1}, E_{2}$ and $E_{3}$ defined in Section 2.3. This requires extending the concept of stochastic strength of a fibre segment to the linear stress concentrations field that occurs near a fibre break (Figure 3).

Consider the stress field after failure of segment $\mathcal{A}_{1}$ in a level-[1] bundle (Figure 3a). Let $X_{\mathrm{U}, \mathrm{e}}^{\mathcal{B}_{2}}$ be the stochastic strength of the fibre segment $\mathcal{B}_{2}$ under uniform stresses (indicated by the subscript $\mathrm{U}$ ); $\mathcal{B}_{2}$ fails if $X_{\mathrm{U}, \mathrm{e}}^{\mathcal{B}_{2}} \leq \sigma^{\infty}$. Similarly, $X_{\mathrm{K}, \mathrm{e}}^{\mathcal{B}_{1}}$ is the stochastic strength of the segment $\mathcal{B}_{1}$ under linear stress concentrations, characterised by a variable remote stress $\sigma^{\infty}$ and constant factor $k ; \mathcal{B}_{1}$ fails if $X_{\mathrm{K}, \mathrm{e}}^{\mathcal{B}_{1}} \leq \sigma^{\infty}$. 
The sequences of events leading to bundle failure $\left(E_{1}, E_{2}\right.$ and $\left.E_{3}\right)$ can now be formally defined as:

$E_{1}$ : Unstable bundle failure at $\sigma^{\infty}=X_{\mathrm{U}, \mathrm{c}}^{[1]}$. All 4 segments survive the uniform stress field for $\sigma^{\infty}<X_{\mathrm{U}, \mathrm{c}}^{[1]} ; \mathcal{A}_{1}$ fails when $\sigma^{\infty}=X_{\mathrm{U}, \mathrm{c}}^{[1]}$, triggering failure of $\mathcal{B}_{1}$ due to stress concentrations. Formally,

$$
E_{1}=\left\{\sigma^{\infty}:\left[X_{\mathrm{U}, \mathrm{e}}^{\mathcal{A}_{1}}=\sigma^{\infty}\right] \wedge\left[X_{\mathrm{U}, \mathrm{e}}^{\mathcal{A}_{2}}>\sigma^{\infty}\right] \wedge\left[X_{\mathrm{U}, \mathrm{e}}^{\mathcal{B}_{1}}>\sigma^{\infty} \wedge X_{\mathrm{K}, \mathrm{e}}^{\mathcal{B}_{1}} \leq \sigma^{\infty}\right] \wedge\left[X_{\mathrm{U}, \mathrm{e}}^{\mathcal{B}_{2}}>\sigma^{\infty}\right]\right\}
$$

$E_{2}$ : Stable bundle failure due to stress concentrations at $\sigma^{\infty}=X_{\mathrm{U}, \mathrm{c}}^{[1]} \cdot \mathcal{A}_{1}$ fails when $\sigma^{\infty}=X_{\mathrm{U}, \mathrm{e}}^{\mathcal{A}}<X_{\mathrm{U}, \mathrm{c}}^{[1]}$, and (from the assumption $\left.\mathbf{A}(\mathrm{ii})\right) \mathcal{A}_{2}$ survives $\sigma^{\infty} \leq X_{\mathrm{U}, \mathrm{e}}^{\mathcal{A}} . \mathcal{B}_{2}$ withstands the stress field for $\sigma^{\infty} \leq X_{\mathrm{U}, \mathrm{c}}^{[1]}$, but $\mathcal{B}_{1}$ fails under stress concentrations at $\sigma^{\infty}=X_{\mathrm{U}, \mathrm{c}}^{[1]}$. Formally,

$$
E_{2}=\left\{\sigma^{\infty}:\left[X_{\mathrm{U}, \mathrm{e}}^{\mathcal{A}_{1}}<\sigma^{\infty}\right] \wedge\left[X_{\mathrm{U}, \mathrm{e}}^{\mathcal{A}_{2}}>X_{\mathrm{U}, \mathrm{e}}^{\mathcal{A}_{1}}\right] \wedge\left[X_{\mathrm{K}, \mathrm{e}}^{\mathcal{B}_{1}}=\sigma^{\infty}\right] \wedge\left[X_{\mathrm{U}, \mathrm{e}}^{\mathcal{B}_{2}}>\sigma^{\infty}\right]\right\}
$$

$E_{3}$ : Stable bundle failure due to independent fibre flaws at $\sigma^{\infty}=X_{\mathrm{U}, \mathrm{c}}^{[1]} \cdot \mathcal{A}_{1}$ fails when $\sigma^{\infty}=X_{\mathrm{U}, \mathrm{e}}^{\mathcal{A}}<X_{\mathrm{U}, \mathrm{c}}^{[1]}$, and $\mathcal{A}_{2}$ survives $\sigma^{\infty} \leq X_{\mathrm{U}, \mathrm{e}}^{\mathcal{A}} \cdot \mathcal{B}_{1}$ withstands the stress concentrations field for $\sigma^{\infty} \leq X_{\mathrm{U}, \mathrm{c}}^{[1]}$, but $\mathcal{B}_{2}$ fails under uniform stresses at $\sigma^{\infty}=X_{\mathrm{U}, \mathrm{c}}^{[1]}$, either due to a stress increment $\mathrm{d} \sigma^{\infty}$ or domain extension $\mathrm{d} l_{\mathrm{e}}\left(\sigma^{\infty}\right)$ (from Equation 6$)$ :

$$
E_{3}=\left\{\sigma^{\infty}:\left[X_{\mathrm{U}, \mathrm{e}}^{\mathcal{A}_{1}}<\sigma^{\infty}\right] \wedge\left[X_{\mathrm{U}, \mathrm{e}}^{\mathcal{A}_{2}}>X_{\mathrm{U}, \mathrm{e}}^{\mathcal{A}_{1}}\right] \wedge\left[X_{\mathrm{K}, \mathrm{e}}^{\mathcal{B}_{1}}>\sigma^{\infty}\right] \wedge\left[X_{\mathrm{U}, \mathrm{e}}^{\mathcal{B}_{2}}=\sigma^{\infty}\right]\right\}
$$

The events above assume the weakest segment in the control length is $\mathcal{A}_{1}$ which (without loss of generality) represents $1 / 4$ of the cases. Therefore, the level-[1] bundle strength distribution within the control length is:

$$
\operatorname{Pr}\left(X_{\mathrm{U}, \mathrm{c}}^{[1]}=\sigma^{\infty}\right)=4 \cdot\left[\operatorname{Pr}\left(E_{1}\right)+\operatorname{Pr}\left(E_{2}\right)+\operatorname{Pr}\left(E_{3}\right)\right]
$$

The probability for each sequence of events $E_{1}, E_{2}$ and $E_{3}$ is defined from the single-fibre strength distributions. All fibre segments have independent and identically distributed strengths; therefore, failure probabilities (cumulative distribution functions) under uniform stresses and under linear stress concentrations are represented respectively as 
$F_{\mathrm{U}, \mathrm{e}}^{[0]}\left(\sigma^{\infty}\right)=\operatorname{Pr}\left(X_{\mathrm{U}, \mathrm{e}}^{\mathcal{B}_{2}} \leq \sigma^{\infty}\right)$ and $F_{\mathrm{K}, \mathrm{e}}^{[0]}\left(\sigma^{\infty}\right)=\operatorname{Pr}\left(X_{\mathrm{K}, \mathbf{e}}^{\mathcal{B}_{1}} \leq \sigma^{\infty}\right)$. Consequently:

$$
\begin{aligned}
& \operatorname{Pr}\left(E_{1}\right) \stackrel{\text { Eq. C.1 }}{=}\left[\mathrm{d} F_{\mathrm{U}, \mathbf{e}}^{[0]}\left(\sigma^{\infty}\right)\right] \cdot\left[1-F_{\mathrm{U}, \mathbf{e}}^{[0]}\left(\sigma^{\infty}\right)\right] \cdot\left[F_{\mathrm{K}, \mathbf{e}}^{[0]}\left(\sigma^{\infty}\right)-F_{\mathrm{U}, \mathbf{e}}^{[0]}\left(\sigma^{\infty}\right)\right] \cdot\left[1-F_{\mathrm{U}, \mathbf{e}}^{[0]}\left(\sigma^{\infty}\right)\right] \\
& \operatorname{Pr}\left(E_{2}\right) \stackrel{\text { Eq. C.2 }}{=} \int_{\sigma=0}^{\sigma^{\infty}}\left(\left[\mathrm{d} F_{\mathrm{U}, \mathbf{e}}^{[0]}(\sigma)\right] \cdot\left[1-F_{\mathrm{U}, \mathbf{e}}^{[0]}(\sigma)\right]\right) \cdot\left[\mathrm{d} F_{\mathrm{K}, \mathbf{e}}^{[0]}\left(\sigma^{\infty}\right)\right] \cdot\left[1-F_{\mathrm{U}, \mathbf{e}}^{[0]}\left(\sigma^{\infty}\right)\right]= \\
& =\left[F_{\mathrm{U}, \mathbf{e}}^{[0]}\left(\sigma^{\infty}\right)\right] \cdot\left[1-F_{\mathrm{U}, \mathbf{e}}^{[0]}\left(\sigma^{\infty}\right) / 2\right] \cdot\left[\mathrm{d} F_{\mathrm{K}, \mathbf{e}}^{[0]}\left(\sigma^{\infty}\right)\right] \cdot\left[1-F_{\mathrm{U}, \mathbf{e}}^{[0]}\left(\sigma^{\infty}\right)\right] \\
& \operatorname{Pr}\left(E_{3}\right) \stackrel{\text { Eq. C.3 }}{=}\left[F_{\mathrm{U}, \mathbf{e}}^{[0]}\left(\sigma^{\infty}\right)\right] \cdot\left[1-F_{\mathrm{U}, \mathbf{e}}^{[0]}\left(\sigma^{\infty}\right) / 2\right] \cdot\left[1-F_{\mathrm{K}, \mathbf{e}}^{[0]}\left(\sigma^{\infty}\right)\right] \cdot\left[\mathrm{d} F_{\mathrm{U}, \mathbf{e}}^{[0]}\left(\sigma^{\infty}\right)\right]
\end{aligned}
$$

The level-[1] bundle strength distribution $F_{\mathrm{U}, \mathrm{c}}^{[1]}\left(\sigma^{\infty}\right)$ is calculated by replacing the probabilities above in Equation C.4, and integrating $\operatorname{Pr}\left(X_{\mathrm{U}, \mathrm{c}}^{[1]}=\sigma\right)$ for all $\sigma \leq \sigma^{\infty}$ :

$$
\begin{aligned}
F_{\mathrm{U}, \mathrm{e}}^{[1]}\left(\sigma^{\infty}\right)=4 \cdot \int_{\sigma=0}^{\sigma^{\infty}} & {\left[\mathrm{d} F_{\mathrm{U}, \mathbf{e}}^{[0]}(\sigma) \cdot\left[1-F_{\mathrm{U}, \mathbf{e}}^{[0]}(\sigma)\right] \cdot\left[F_{\mathrm{K}, \mathbf{e}}^{[0]}(\sigma)-F_{\mathrm{U}, \mathbf{e}}^{[0]}(\sigma)\right] \cdot\left[1-F_{\mathrm{U}, \mathbf{e}}^{[0]}(\sigma)\right]+\right.} \\
& +F_{\mathrm{U}, \mathbf{e}}^{[0]}(\sigma) \cdot\left[1-F_{\mathrm{U}, \mathbf{e}}^{[0]}(\sigma) / 2\right] \cdot \mathrm{d} F_{\mathrm{K}, \mathbf{e}}^{[0]}(\sigma) \cdot\left[1-F_{\mathrm{U}, \mathbf{e}}^{[0]}(\sigma)\right]+ \\
& \left.+F_{\mathrm{U}, \mathbf{e}}^{[0]}(\sigma) \cdot\left[1-F_{\mathrm{U}, \mathbf{e}}^{[0]}(\sigma) / 2\right] \cdot\left[1-F_{\mathrm{K}, \mathbf{e}}^{[0]}(\sigma)\right] \cdot \mathrm{d} F_{\mathrm{U}, \mathbf{e}}^{[0]}(\sigma)\right]
\end{aligned}
$$

Recalling that $F^{N} \mathrm{~d} F=\mathrm{d}\left(F^{N+1}\right) /(N+1)$ and re-arranging the equation (omitting the integration variable $\sigma$ and the single-fibre superscript [0] for readability) yields:

$$
\begin{aligned}
& F_{\mathrm{U}, \mathrm{c}}^{[1]}\left(\sigma^{\infty}\right)=-F_{\mathrm{U}, \mathrm{e}}\left(\sigma^{\infty}\right)^{4}+2 \cdot F_{\mathrm{U}, \mathrm{e}}\left(\sigma^{\infty}\right)^{3}+2 \cdot\left[\int F_{\mathrm{K}, \mathrm{e}} \mathrm{d}\left(F_{\mathrm{U}, \mathrm{e}}^{3}\right)+\int F_{\mathrm{U}, \mathrm{e}}^{3} \mathrm{~d} F_{\mathrm{K}, \mathrm{e}}\right]_{\sigma=0}^{\sigma^{\infty}}+ \\
& -6 \cdot\left[\int F_{\mathrm{K}, \mathrm{e}} \cdot\left(F_{\mathrm{U}, \mathrm{e}}^{2}\right)+\int F_{\mathrm{U}, \mathrm{e}}^{2} \mathrm{~d} F_{\mathrm{K}, \mathrm{e}}\right]_{\sigma=0}^{\sigma^{\infty}}+4 \cdot\left[\int F_{\mathrm{K}, \mathrm{e}} \mathrm{d} F_{\mathrm{U}, \mathrm{e}}+\int F_{\mathrm{U}, \mathrm{e}} \mathrm{d} F_{\mathrm{K}, \mathrm{e}}\right]_{\sigma=0}^{\sigma^{\infty}}
\end{aligned}
$$

Integrating by parts each pair of integrals results into:

$$
\begin{aligned}
F_{\mathrm{U}, \mathrm{c}}^{[1]}\left(\sigma^{\infty}\right)=-F_{\mathrm{U}, \mathbf{e}}^{[0]}\left(\sigma^{\infty}\right)^{4}+2 \cdot F_{\mathrm{U}, \mathbf{e}}^{[0]} & \left(\sigma^{\infty}\right)^{3}+2 \cdot F_{\mathrm{U}, \mathbf{e}}^{[0]}\left(\sigma^{\infty}\right)^{3} \cdot F_{\mathrm{K}, \mathbf{e}}^{[0]}\left(\sigma^{\infty}\right)+ \\
& -6 \cdot F_{\mathrm{U}, \mathbf{e}}^{[0]}\left(\sigma^{\infty}\right)^{2} \cdot F_{\mathrm{K}, \mathbf{e}}^{[0]}\left(\sigma^{\infty}\right)+4 \cdot F_{\mathrm{U}, \mathbf{e}}^{[0]}\left(\sigma^{\infty}\right) \cdot F_{\mathrm{K}, \mathbf{e}}^{[0]}\left(\sigma^{\infty}\right)
\end{aligned}
$$

Converting all distributions to survival probabilities, $S\left(\sigma^{\infty}\right)=1-F\left(\sigma^{\infty}\right)$, this corresponds to the scaling law proposed in Equation 8 of Section 2.3. 


\section{Appendix D. Proof of asymptotic behaviour for strength distributions}

\section{Appendix D.1. Right tail asymptote}

Assuming single-fibre strength follows a Weibull distribution (thus $\ln \left[S_{\mathrm{K}, \mathrm{r}}^{[0]}\right]=\mathcal{C}_{\mathrm{K}} \ln \left[S_{\mathrm{U}, \mathrm{r}}^{[0]}\right]$ from Equation B.7), applying the scaling law (Equation 14) to $i=0$ yields:

$$
\begin{aligned}
& \ln \left(S_{\mathrm{U}, \mathrm{r}}^{[1]}\right)=2 \cdot \ln \left(S_{\mathrm{U}, \mathrm{r}}^{[0]}\right)+\frac{l_{\mathrm{r}}}{2 \cdot l_{\mathrm{e}}^{[0]}} \cdot \ln \left(1+2 \cdot\left[S_{\mathrm{U}, \mathrm{r}}^{[0]}\right]^{\left(\mathcal{C}_{\mathrm{K}}-3\right) \cdot \cdot_{\frac{\mathrm{e}}{l_{\mathrm{r}}}}^{[0]}}-2 \cdot\left[S_{\mathrm{U}, \mathrm{r}}^{[0]}\right]^{\left(\mathcal{C}_{\mathrm{K}}-1\right) \cdot \cdot_{\frac{\mathrm{e}}{l_{\mathrm{r}}}}^{[0]}}\right)= \\
& =\frac{\left(1+\mathcal{C}_{\mathrm{K}}\right) \cdot \ln \left(S_{\mathrm{U}, \mathrm{r}}^{[0]}\right)}{2}+\frac{l_{\mathrm{r}}}{2 \cdot l_{\mathrm{e}}^{[0]}} \cdot \ln \left(2+\left[S_{\mathrm{U}, \mathrm{r}}^{[0]}\right]^{\left(3-\mathcal{C}_{\mathrm{K}} \cdot \cdot^{\left[\frac{[0]}{l_{\mathrm{r}}}\right.}\right.}-2 \cdot\left[S_{\mathrm{U}, \mathrm{r}}^{[0]}\right]^{2 \cdot \cdot_{\frac{l_{\mathrm{r}}}{l_{\mathrm{r}}}}^{[0]}}\right)
\end{aligned}
$$

For large stresses, $S_{\mathrm{U}, \mathrm{r}}^{[0]}\left(\sigma^{\infty}\right) \rightarrow 0$ and $l_{\mathrm{e}}^{[0]} \rightarrow \infty$ (Equations 6 and 9 ), so the contribution of the terms highlighted above vanishes (as long as exponents are positive). Therefore,

$$
\begin{aligned}
& \lim _{\sigma^{\infty} \rightarrow \infty} \ln \left[S_{\mathrm{U}, \mathbf{r}}^{[1]}\left(\sigma^{\infty}\right)\right]=\psi \cdot \ln \left[S_{\mathrm{U}, \mathrm{r}}^{[0]}\left(\sigma^{\infty}\right)\right] \Rightarrow \lim _{\sigma^{\infty} \rightarrow \infty} S_{\mathrm{U}, \mathbf{r}}^{[1]}\left(\sigma^{\infty}\right)=\exp \left[-\psi \cdot\left(\frac{\sigma^{\infty}}{\sigma_{0}^{\mathbf{f}}}\right)^{m}\right], \\
& \text { with } \quad \psi= \begin{cases}2 & , \mathcal{C}_{\mathrm{K}} \geq 3 \\
\frac{1+\mathcal{C}_{\mathrm{K}}}{2} & , \mathcal{C}_{\mathrm{K}}<3\end{cases}
\end{aligned}
$$

The right tail asymptote of $S_{\mathrm{U}, \mathrm{r}}^{[1]}$ is recognisably a Weibull distribution. Consequently, the same argument can be applied recursively up to any level- $[i]$ bundle, whose right tail will then tend asymptotically to a Weibull distribution as well ( $\psi$ as defined above):

$$
\lim _{\sigma^{\infty} \rightarrow \infty} \ln \left[S_{\mathrm{U}, \mathrm{r}}^{[i]}\left(\sigma^{\infty}\right)\right]=\psi \cdot \ln \left[S_{\mathrm{U}, \mathrm{r}}^{[i-1]}\left(\sigma^{\infty}\right)\right] \Rightarrow \lim _{\sigma^{\infty} \rightarrow \infty} S_{\mathrm{U}, \mathrm{r}}^{[i]}\left(\sigma^{\infty}\right)=\exp \left[-\psi^{i} \cdot\left(\frac{\sigma^{\infty}}{\sigma_{0}^{\mathrm{f}}}\right)^{m}\right]
$$

\section{Appendix D.2. Left tail asymptote}

The single-fibre survival probabilities at the effective recovery length can be expressed as (Equations 6, 9 and 10):

$$
S_{\mathrm{U}, \mathrm{e}}^{[0]}\left(\sigma^{\infty}\right)=\exp \left(-u^{[0]}\right) \quad \text { and } \quad S_{\mathrm{K}, \mathbf{e}}^{[0]}\left(\sigma^{\infty}\right)=\exp \left(-\mathcal{C}_{\mathrm{K}} \cdot u^{[0]}\right), \quad \text { with } \quad u^{[0]}=\frac{l_{\mathrm{e}}^{[0]}}{l_{\mathrm{r}}} \cdot\left(\frac{\sigma^{\infty}}{\sigma_{0}^{\mathrm{f}}}\right)^{m}
$$

According to the scaling law in Equation 8, the level-[1] survival probability in the control length becomes: 


$$
S_{\mathrm{U}, \mathrm{c}}^{[1]}\left(\sigma^{\infty}\right)=\exp \left[-4 \cdot u^{[0]}\right]+2 \cdot \exp \left[-\left(1+\mathcal{C}_{\mathrm{K}}\right) \cdot u^{[0]}\right]-2 \cdot \exp \left[-\left(3+\mathcal{C}_{\mathrm{K}}\right) \cdot u^{[0]}\right]
$$

Taking the limit for low stresses, and using the $2^{\text {nd }}$ order Taylor expansion of the exponential function $-\lim _{\psi \rightarrow 0} \exp (\psi)=1+\psi+\psi^{2} / 2$ - then:

$$
\begin{aligned}
\lim _{\sigma^{\infty} \rightarrow 0} S_{\mathrm{U}, \mathrm{c}}^{[1]}\left(\sigma^{\infty}\right) & =\left(1-4 \cdot u^{[0]}+\left[4 \cdot u^{[0]}\right]^{2} / 2\right)+2 \cdot\left(1-\left(1+\mathcal{C}_{\mathrm{K}}\right) \cdot u^{[0]}+\left[\left(1+\mathcal{C}_{\mathrm{K}}\right) \cdot u^{[0]}\right]^{2} / 2\right) \\
& -2 \cdot\left(1-\left(3+\mathcal{C}_{\mathrm{K}}\right) \cdot u^{[0]}+\left[\left(3+\mathcal{C}_{\mathrm{K}}\right) \cdot u^{[0]}\right]^{2} / 2\right)=1-4 \cdot \mathcal{C}_{\mathrm{K}} \cdot\left(u^{[0]}\right)^{2}= \\
& =\lim _{\sigma^{\infty} \rightarrow 0} \exp \left[-4 \cdot \mathcal{C}_{\mathrm{K}} \cdot\left(u^{[0]}\right)^{2}\right]=\lim _{\sigma^{\infty} \rightarrow 0} \exp \left[-4 \cdot \mathcal{C}_{\mathrm{K}} \cdot\left(\frac{l_{\mathrm{e}}^{[0]}}{l_{\mathrm{r}}}\right)^{2} \cdot\left(\frac{\sigma^{\infty}}{\sigma_{0}^{\mathrm{f}}}\right)^{2 \cdot m}\right]
\end{aligned}
$$

Scaling from $l_{\mathrm{c}}^{[1]}$ (Equation 7 ) to the reference length (WLT as in Equation 1),

$$
\lim _{\sigma^{\infty} \rightarrow 0} S_{\mathrm{U}, \mathrm{r}}^{[1]}\left(\sigma^{\infty}\right)=\exp \left[-2 \cdot \mathcal{C}_{\mathrm{K}} \cdot \frac{l_{\mathrm{e}}^{[0]}}{l_{\mathrm{r}}} \cdot\left(\frac{\sigma^{\infty}}{\sigma_{0}^{\mathrm{f}}}\right)^{2 \cdot m}\right]=\exp \left[-4 \cdot \mathcal{C}_{\mathrm{K}} \cdot \frac{A^{\mathrm{f}} \cdot \sigma_{0}^{\mathrm{f}}}{C^{[0]} \cdot \tau_{\mathrm{SL}}} \cdot\left(\frac{\sigma^{\infty}}{\sigma_{0}^{\mathrm{f}}}\right)^{2 \cdot m+1}\right]
$$

This shows that the left tail asymptote of a level-[1] strength distribution preserves the Weibull nature of the single-fibre distribution (although with different parameters). Consequently, the same argument can be applied recursively up to any level- $[i]$ bundle, hence its left tail Weibull parameters $\left(m_{\text {LTA }}^{[i]}\right.$ and $\left.\sigma_{0, \mathrm{LTA}}^{[i]}\right)$ are related to those of a level$[i-1]$ bundle by:

$$
\begin{aligned}
& -1] \text { bundle by: } \\
& m_{\mathrm{LTA}}^{[i]}=2 \cdot m_{\mathrm{LTA}}^{[i-1]}+1 \quad \text { and } \quad \sigma_{0, \mathrm{LTA}}^{[i]}=\sigma_{0, \mathrm{LTA}}^{[i-1]} \cdot\left(4 \cdot \mathcal{C}_{\mathrm{K}, \mathrm{LTA}}^{[i-1]} \cdot \frac{n^{[i-1]} \cdot A^{\mathrm{f}} \cdot \sigma_{0, \mathrm{LTA}}^{[i-1]}}{C^{[i-1]} \cdot \tau_{\mathrm{SL}} \cdot l_{\mathrm{r}}}\right)^{-1 / m_{\mathrm{LTA}}^{[i]}}, \\
& \text { with } \quad \mathcal{C}_{\mathrm{K}, \mathrm{LTA}}^{[i]}=\frac{k^{m_{\mathrm{LTA}}^{[i]}+1}-1}{\left(m_{\mathrm{LTA}}^{[i]}+1\right) \cdot(k-1)} \quad \text { and } \quad m_{\mathrm{LTA}}^{[0]}=m, \quad \sigma_{0, \mathrm{LTA}}^{[0]}=\sigma_{0}^{\mathrm{f}} \quad(\mathrm{D} .8)
\end{aligned}
$$

\section{References}

Accorsi, M.L., Pegoretti, A., Dibenedetto, A.T., 1996. Dynamic analysis of fibre breakage in single- and multiple-fibre composites. Journal of Materials Science 31, 41814187.

Bažant, Z.P., 1999. Size effect on structural strength: a review. Archive of Applied Mechanics 69, 703-725. 
Bažant, Z.P., Pang, S.D., 2007. Activation energy based extreme value statistics and size effect in brittle and quasibrittle fracture. Journal of the Mechanics and Physics of Solids 55, 91-131.

Beetz, C.P., 1982. The analysis of carbon-fiber strength distributions exhibiting multiple-modes of failure. Fibre Science and Technology 16, 45-59.

Behzadi, S., Curtis, P.T., Jones, F.R., 2009. Improving the prediction of tensile failure in unidirectional fibre composites by introducing matrix shear yielding. Composites Science and Technology 69, 2421-2427.

Beyerlein, I.J., Phoenix, S.L., 1996a. Statistics for the strength and size effects of microcomposites with four carbon fibers in epoxy resin. Composites Science and Technology 56, 75-92.

Beyerlein, I.J., Phoenix, S.L., 1996b. Stress concentrations around multiple fiber breaks in an elastic matrix with local yielding or debonding using quadratic influence superposition. Journal of the Mechanics and Physics of Solids 44, 1997-2039.

Carpinteri, A., 1994. Scaling laws and renormalization-groups for strength and toughness of disordered materials. International Journal of Solids and Structures 31, 291302.

Curtin, W.A., 1991. Theory of mechanical properties of ceramic-matrix composites. Journal of the American Ceramics Society 74, 2837-2845.

Curtin, W.A., 1993. The tough to brittle transition in brittle matrix composites. Journal of the Physics and Mechanics of Solids 41, 217-245.

Curtin, W.A., 1999. Stochastic damage evolution and failure in fiber-reinforced composites. Advances in Applied Mechanics 36, 163-253.

Curtin, W.A., Takeda, N., 1998. Tensile strength of fiber-reinforced composites: I. Model and effects of local fiber geometry. Journal of composite materials 32, 20422059.

Curtis, P.S., 1986. A computer model of the tensile failure process in unidirectional fibre composites. Composites Science and Technology 27, 63-86. 
Daniels, H.E., 1945. The statistical theory of the strength of bundles of threads. I. Proceedings of the Royal Society A - Mathematical, Physical and \& Engineering Science 183, 405-435.

Dow Plastics, 1999. Dow liquid epoxy resins. Form No. 296-00224-0199 WC+M. http: //epoxy.dow.com/epoxy/tech/index.htm, last accessed on 29 January 2011.

Fukuda, H., Kawata, K., 1977. On the strength distribution of unidirectional fibre composites. Fibre Science and Technology 10, 53-63.

Gulino, R., Schwartz, P., Phoenix, S., 1991. Experiments on shear deformation, debonding and local load transfer in a model graphite/glass/epoxy microcomposite. Journal of Materials Science 26, 6655-6672.

Harlow, D.G., Phoenix, S.L., 1978a. The chain-of-bundles probability model for the strength of fibrous materials. I: Analysis and conjectures. Journal of Composite Materials 12, 195-214.

Harlow, D.G., Phoenix, S.L., 1978b. The chain-of-bundles probability model for the strength of fibrous materials. II: A numerical study of convergence. Journal of Composite Materials 12, 314-334.

Harper, L.T., Turner, T.A., Warrior, N.A., Rudd, C.D., 2007. Characterisation of random carbon fibre composites from a directed fibre preforming process: The effect of tow filamentisation. Composites Part A - Applied Science and Manufacturing $38,755-770$.

Hedgepeth, J.M., 1961. Stress concentrations in filamentary structures. Technical Report TN D 882. NASA. Washington DC, USA.

Hobbiebrunken, T., Fiedler, B., Hojo, M., Tanaka, M., 2007. Experimental determination of the true epoxy resin strength using micro-scaled specimens. Composites Part A - Applied Science and Manufacturing 38, 814-818.

Kazanci, M., 2004. Carbon fiber reinforced microcomposites in two different epoxies. Polymer Testing 23, 747-753.

Kelly, A., Tyson, W., 1965. Tensile properties of fibre-reinforced metals: Copper/tungsten and copper/molybdenum. Journal of the Mechanics and Physics of Solids 13, 329-338. 
Laffan, M.J., Pinho, S.T., Robinson, P., Iannucci, L., 2010. Measurement of the in situ ply fracture toughness associated with mode I fibre tensile failure in FRP. Part II: Size and lay-up effects. Composites Science and Technology 70, 614-621.

Landis, C.M., Beyerlein, I.J., McMeeking, R.M., 2000. Micromechanical simulation of the failure of fiber reinforced composites. Journal of the Physics and Mechanics of Solids 48, 621-648.

Landis, C.M., McMeeking, R.M., 1999. A shear-lag model for a broken fiber embedded in a composite with a ductile matrix. Composites Science and Technology 59, 447457.

Mahesh, S., Phoenix, S.L., Beyerlein, I.J., 2002. Strength distributions and size effects for $2 \mathrm{D}$ and $3 \mathrm{D}$ composites with weibull fibers in an elastic matrix. International Journal of Fracture 115, 41-85.

de Morais, A.B., 2001. Stress distribution along broken fibres in polymer-matrix composites. Composites Science and Technology 61, 1571-1580.

de Morais, A.B., 2006. Prediction of the longitudinal tensile strength of polymer matrix composites. Composites Science and Technology 66, 2990-2996.

Nairn, J.A., 1997. On the use of shear-lag methods for analysis of stress transfer unidirectional composites. Mechanics of Materials 26, 63-80.

Nedele, M.R., Wisnom, M.R., 1994. 3-dimensional finite-element analysis of the stressconcentration at a single-fiber break. Composites Science and Technology 51, 517524.

Newman, W.I., Gabrielov, A.M., 1991. Failure of hierarchical distributions of fiberbundles. 1. International Journal of Fracture 50, 1-14.

Okabe, T., Takeda, N., 2001. A 3D shear-lag model considering micro-damage and statistical strength prediction of unidirectional fiber-reinforced composites. Composites Science and Technology 61, 1773-1787.

Okabe, T., Takeda, N., 2002. Size effect on tensile strength of unidirectional CFRP composites - experiment and simulation. Composites Science and Technology 62, 2053-2064. 
Phoenix, S.L., Ibnabdeljalil, M., Hui, C.Y., 1997. Size effects in the distribution for strength of brittle matrix fibrous composites. International Journal of Solids and Structures 34, 545-568.

Phoenix, S.L., Schwartz, P., Robinson, H.H., 1988. Statistics for the strength and lifetime in creep-rupture of model carbon epoxy composites. Composites Science and Technology 32, 81-120.

Phoenix, S.L., Smith, R.L., 1983. A comparison of probabilistic techniques for the strength of fibrous materials under local load-sharing among fibers. International Journal of Solids and Structures 19, 479-496.

Pimenta, S., Pinho, S.T., 2013. An analytical model for the translaminar tensile toughness of fibre composites with stochastic fractal fracture surfaces. To be submitted to the Journal of the Mechanics and Physics of Solids .

Pimenta, S., Pinho, S.T., Robinson, P., Wong, K.H., Pickering, S.J., 2010. Mechanical analysis and toughening mechanisms of a multiphase recycled CFRP. Composites Science and Technology 70, 1713-1725.

Pradhan, S., Hansen, A., Chakrabarti, B.K., 2010. Failure processes in elastic fiber bundles. Reviews of Modern Physics 82, 499-555.

Scott, A.E., Mavrogordato, M., Wright, P., Sinclair, I., Spearing, S.M., 2011. In situ fibre fracture measurement in carbon-epoxy laminates using high resolution computed tomography. Composites Science and Technology 71, 1471-1477.

Sihn, S., Kim, R.Y., Kawabe, K., Tsai, S.W., 2007. Experimental studies of thin-ply laminated composites. Composites Science and Technology 67, 996-1008.

Stoner, E.G., Edie, D.D., Durham, S.D., 1994. An end-effect model for the single-filament tensile test. Journal of Materials Science 29, 6561-6574.

Swolfs, Y., Gorbatikh, L., Romanov, V., Orlova, S., Lomov, S.V., Verpoest, I., 2013. Stress concentrations in an impregnated fibre bundle with random fibre packing. Composites Science and Technology 74, 113-120.

Wada, A., Fukuda, H., 1999. Approximate upper and lower bounds for the strength of unidirectional composites. Composites Science and Technology 59, 89-95. 
Weibull, G.W., 1951. A statistical distribution function of wide applicability. Journal of Applied Mathematics , 293-297.

Wisnom, M.R., 1999. Size effects in the testing of fibre-composite materials. Composites Science and Technology 59, 1937-1957.

Wisnom, M.R., Green, D., 1995. Tensile failure due to interaction between fibre breaks. Composites 26, 499-508.

Wisnom, M.R., Hallett, S.R., Soutis, C., 2010. Scaling effects in notched composites. Journal of Composite Materials 44, 195-210.

Zhandarov, S., Pisanova, E., Lauke, B., 1998. Is there any contradiction between the stress and energy failure criteria in micromechanical tests? Part I. Crack initiation: stress-controlled or energy-controlled? Composite Interfaces 5, 387-404. 